TI 2015-064/I

Tinbergen Institute Discussion Paper

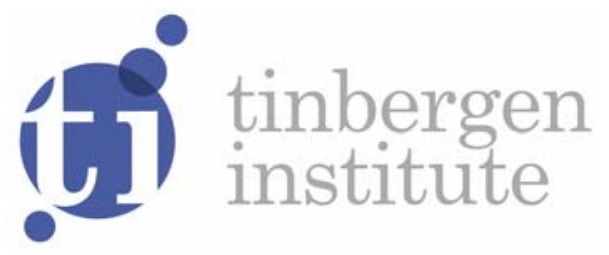

\title{
Homothetic Efficiency and Test Power: A Non-Parametric Approach
}

\author{
Jan Heufer \\ Per Hjertstrand ${ }^{2}$
}

' Erasmus School of Economics, Erasmus University Rotterdam, and Tinbergen Institute, the Netherlands;

2 Research Institute of Industrial Economics (IFN), Sweden. 
Tinbergen Institute is the graduate school and research institute in economics of Erasmus University Rotterdam, the University of Amsterdam and VU University Amsterdam.

More TI discussion papers can be downloaded at http://www.tinbergen.nl

Tinbergen Institute has two locations:

Tinbergen Institute Amsterdam

Gustav Mahlerplein 117

1082 MS Amsterdam

The Netherlands

Tel.: +31(0)205251600

Tinbergen Institute Rotterdam

Burg. Oudlaan 50

3062 PA Rotterdam

The Netherlands

Tel.: +31(0)10 4088900

Fax: $+31(0) 104089031$

Duisenberg school of finance is a collaboration of the Dutch financial sector and universities, with the ambition to support innovative research and offer top quality academic education in core areas of finance.

DSF research papers can be downloaded at: http://www.dsf.nl/

Duisenberg school of finance

Gustav Mahlerplein 117

1082 MS Amsterdam

The Netherlands

Tel.: +31(0)20 5258579 


\title{
Homothetic Efficiency and Test Power: A Non-Parametric Approach*
}

\author{
Jan Heufer ${ }^{\mathrm{a}}$ and Per Hjertstrand ${ }^{\mathrm{b}}$ \\ ${ }^{a}$ Erasmus School of Economics and Tinbergen Institute, Rotterdam \\ ${ }^{\mathrm{b}}$ Research Institute of Industrial Economics, Stockholm
}

31 May 2015

\begin{abstract}
We provide a nonparametric revealed preference approach to demand analysis based on homothetic efficiency. Homotheticity is a useful restriction but data rarely satisfies testable conditions. To overcome this we provide a way to estimate homothetic efficiency of consumption choices. It generalises Heufer's (2013) two-dimensional concept to arbitrary dimensions and is motivated by a form of rationalisation similar to Halevy et al. (2012). The method allows to recover larger preferred and worse sets and provides considerably more preditive success and discriminatory power against random behaviour. An application to experimental and household expenditure data illustrates the potential of our approach.
\end{abstract}

JEL classification: C14; D11; D12.

Keywords: Demand Theory; Efficiency; Experimental Economics; Homotheticity; Non-parametric Analysis; Revealed Preference; Utility Maximisation.

\footnotetext{
${ }^{*}$ Corresponding author: Per Hjertstrand. ${ }^{a}$ Erasmus School of Economics and Tinbergen Institute, Erasmus University, 3000 DR Rotterdam, The Netherlands. E-mail: heufer@ese.eur.nl. ${ }^{b}$ Research Institute of Industrial Economics, Box 5565, SE-102, Stockholm, Sweden. Email: per.hjertstrand@ifn.se. Views expressed represent exclusively the authors' own opinions. Access to data collected by Raymond Fisman, Shachar Kariv, and Daniel Markovits is gratefully acknowledged. Thanks to Linda Hirt-Schierbaum for helpful comments.
} 


\section{Introduction}

Homotheticity of consumer preferences is an important and useful concept in both theoretical and empirical work. If a consumer's preferences are homothetic, we can deduce his entire preference relation from a single indifference set. It therefore allows to recover much more of the preferences from a limited data set. Furthermore, testing data for homothetic utility maximisation can provide substantially stronger discriminatory power against alternative hypothesis than testing for utility maximisation alone. Homotheticity has important implications in many different fields of economics; for examples, aggregation of consumer demand and the existence of "community indifference curves", modelling of separable preference structures and its connection to two-stage budgeting, and as a common assumption in the international trade literature.

\subsection{Summary of Contributions}

The purpose of this article is to provide measures for the homothetic efficiency of a data set. We introduce the Homothetic Efficiency Index (HeI) which is a homothetic analogue to the well known Afriat Efficiency Index (AEI, also known as the Critical Cost Efficiency Index, CCEI) which can be interpreted as a measure of wasted income. The HeI generalises the index proposed by Heufer (2013) for the two-dimensional case. We also extend this measure by introducing the Homothetic Efficiency Vector (HEv) which provides efficiency indices for each observed choice and allows for a more detailed and robust data analysis.

Varian's (1983) Homothetic Axiom of Revealed Preference (HARP) can be easily tested with a set of data. It is a necessary and sufficient condition for consistency with homothetic utility maximisation and therefore characterises the hypothesis of homothetic preferences. However, it is a binary test: Either the data satisfy HARP or not. When HARP is violated, the measures introduced here show how close the data come to being consistent with HARP. Both the HEI and the HEv provide the minimal adjustments which are required to make a data set consistent with homothetic utility maximisation. The measures are motivated by $e$ - and h-rationalisation which is similar to a concept recently introduced by Halevy et al. (2012). As HARP is a rather strong condition, it is often violated; without efficiency measures the only conclusion is then that the data is not perfectly consistent. Our measures allow us to go further by quantifying and interpreting the extent of the inconsistency.

We show how the HEv can be used to recover more about a consumers' preference relation when a set of data comes reasonably close to homotheticity. This extends Varian's (1982) and Knoblauch's (1993) approach to non-parametric recoverability of preferences to a situation where data can be assumed to be the result of 
homothetic utility maximisation with minor errors.

A somewhat similar approach was taken by Blundell et al. (2003, 2008), who combined revealed preference conditions with expansion paths estimated using pooled household data. While their approach is indeed powerful, our approach has several advantages. It lends itself to a very useful power analysis that does not require much effort. It focusses on individuals and allows a very detailed analysis of individual preferences. We do not need to pool data to estimate expansion paths and do not require any assumptions about preference homogeneity or adjustments for demographic characteristics. It can be applied equally well to household panel data and experimental data. Besides these advantages, there is still the point that homotheticity is a common - often only implicit - assumption. It should be tested whenever possible but rarely is - in fact, the assumption is often not even discussed. Our contribution makes it very easy for researchers to find out how consistent their data are with homotheticity. Our efficiency index allows to settle the debate of how plausible the assumption of homotheticity is - or rather, it allows to separate the cases where the assumption is justified from cases where it is not.

To illustrate and motivate the methods put forward in this paper we apply them to two data sets. The first application is to data from an experimental dictator game conducted by Fisman et al. (2007). Using this data, we show how our methods can recover detailed information about subjects' preferences. The second application is to a panel of expenditures on non-durable consumption categories for 3,134 Spanish households. With this data, previously analysed in e.g. Browning and Collado (2001), Crawford (2010) and Cherchye et al. (2015), we show that homothetic efficiency can be very high and still have considerably higher discriminatory power against irrational behaviour than standard utility maximisation. We also implement a recent idea by Beatty and Crawford (2011) that combines efficiency and power into a single measure, called predictive success. We find that homothetic utility maximisation is considerably more successful in explaining the demand behaviour of the households than standard utility maximisation.

\subsection{Implications and Applications}

The main empirical applications we see for our approach are

- a better way to test data for consistency with homotheticity;

- an increase of test power, with the potential of making formerly questionable data useful;

- an improvement of bounds on revealed preferred and worse sets and the money metric utility function which facilitates their practical use. 


\section{Testing and Test Power}

Non-parametric tests for homotheticity in consumption and production theory have been considered in the literature before. Varian (1983) introduced HARP, an easily testable axiom that characterises monotonic and convex homothetic utility maximisation. Liu and Wong (2000) provided a stronger testable condition, which characterises strictly convex homothetic utility maximisation.

Testing data for consistency with Varian's (1982) Generalised Axiom of Revealed Preference (GARP), which is a necessary and sufficient condition for non-satiated utility maximisation, is a binary as the test for HARP. The data will either satisfy GARP or not. In cases where the data does not satisfy GARP, studies usually report an efficiency measure such as the AEI combined with various power measures. The most frequently used power measure was introduced by Bronars (1987) who suggested using Monte Carlo methods to compute the power of a test for GARP. One can generate many sets of random choices, usually from a uniform distribution on the budgets, and test these sets for consistency with GARP. The percentage of sets which do not satisfy GARP is the approximate test power.

One problem with the revealed preference approach is that the test power is sometimes very low. This is particuarly true when we wish to allow for small errors in decision making or for measurement error. For example, we might deem an AEI of 0.95 to be acceptable, but allowing for this extent of errors can lead to such a low power that the empirical analysis becomes almost meaningless. HARP is a stronger condition than GARP, and we can expect that it is far less likely that a set of random choices satisfies HARP. However, HARP will often also not be satisfied by the real data. ${ }^{1}$ But homothetic efficiency can be very high for consumer choice data, as we demonstrate in the empirical part of the paper. Thus, homothetic efficiency may provide for an empirical analysis that has substantial discriminatory power against alternative hypothesis such as random behaviour, even when this is not the case for standard efficiency, making previously useless data useful.

This is strongly supported by the results from our empirical applications. Specifically, our main results can be summarized as follows: (i) Efficiency can be very high for HARP, thus providing motivation to assume homothetic preferences. (ii) HARP has considerably higher power than GARP for consumer choice data. For example, while the power of GARP can be below 10 percent for consumer choice data, the power of HARP is close to 100 percent. (iii) Adjusting expenditure for homothetic efficiency has negligible effects on the power. Thus, HaRP can have substantially higher power than GARP even when expenditure is adjusted for efficiency. (iv) Based on the measure of predictive success, homotheticity is a more

\footnotetext{
${ }^{1}$ Manser and McDonald (1988) is a notable exception. They analyse U.S. consumption data from 1959 to 1985 on 101 commondities and find that homothetic preferences are consistent with this data.
} 
suitable model than standard utility maximisation to explain demand behaviour of households in our survey data application.

Using Heufer's (2014a) method we also show that computer generated random data sets which satisfy GARP are very unlikely to satisfy HARP. This shows that HARP adds substantial test power.

\section{Recoverability, Money Metric, and Parametric Estimates}

Varian (1982) described in detail the ways in which a researcher can recover everything that can be said about a consumer's preference based on a finite set of consumption data (see also Knoblauch 1992). Knoblauch (1993) extended Varian's approach to homothetic recoverability. Assuming homotheticity of preferences, if justified, allows the researcher to recover more information about the consumer's preferences.

Our approach allows for this extended recoverability even when homotheticity is violated by providing a way to adjust the data accordingly; with high homothetic efficiency, only minor adjustments are necessary. Our empirical application demonstrates the usefulness of this approach by showing examples of revealed preferred and worse sets of subjects using data from the experiment carried out by Fisman et al. (2005). We show how a simple graphical analysis provides substantial information about the preferences of subjects - Figure 4 in Section 4.2 shows a few examples that demonstrate how much more information about preferences can be recovered using our approach.

Heufer (2014b) introduced a method to compare the risk aversion of two investors using only revealed preference relation. A similar approach to interpersonal comparisons was used by Becker et al. (2013a,b) to compare the strength of the sense of justice of two individuals. These revealed preference approaches to comparability of preferences can be improved if they are based on homothetic revealed preferred and worse sets.

Empirical work often focusses on estimating parameters of particular functional forms corresponding to homothetic utility functions, or demand systems (CobbDouglas and CES are two notable examples which are widely used in the literature). If the estimated utility function is homothetic, testing the data for consistency with HARP and computing efficiency measures can be employed as a robustness check or screening device. Consequently, it may be advisable not to estimate parameters in homothetic demand systems using data with low homothetic efficiency.

Recently, Halevy et al. (2012) introduced methods for parametric recoverability, where parameters are not estimated by minimising a statistical loss function but rather by maximising the money metric utility of a consumer, which is bounded by the revealed preference relation implicit in his set of choices. As the extended homothetic recoverability we propose can lead to tighter bounds on the money 
metric utility function of a consumer, it allows for a better estimation of parameters, in particular those of homothetic utility functions.

\section{Other Applications}

Eisenberg (1961) showed that homotheticity of utility is a sufficient condition for the existence of "community indifference curves" or "average preferences" (see also Gorman 1953). Thus, testing data for how close they come to homotheticity is easily motivated by the important implications for aggregation. See also Chipman (1965) for a discussion of demand aggregation in trade theory, Chipman (1974), Mantel (1976), Polemarchakis (1983), and Varian (1984b) on further issues of aggregation and homotheticity, and Shafer and Sonnenschein (1982) for a survey on market demand.

Gorman (1959) showed that, in general, homothetic separability (i.e., when subutility functions are homothetic) is a sufficient condition for two-stage budgeting. Thus, our methods can be used as pre-tests to check whether homothetic separability is a plausible assumption.

Theories of international trade typically assume that consumers have homothetic preferences (see e.g. Krugman 1980, Melitz 2003, Helpman et al. 2008, Chaney 2008). Thus, our methods can be used to test the underlying assumptions in these models.

Hanoch and Rothschild (1972) and Varian (1984a) described non-parametric ways to test production for homotheticity. ${ }^{2}$ Because this theory is very similar to testing for homotheticity in a consumer choice setting, our methods can be easily adapted to calculate homothetic efficiency of allocating factor inputs in production, and also recover detailed information about the underlying production technology. Finally, it is important to note that homothetic production functions, such as Cobb-Douglas and CES, are extensively used in, for example, empirical macroeconomics when modelling the economy's underlying production technology.

\subsection{Outline}

The rest of the paper is organised as follows. Section 2 introduces the notation, recalls basic revealed preference theory and the non-parametric analysis based on Varian (1982) and, for the particular case of homotheticity, the contribution of Knoblauch (1993). Section 3 introduces the concept of homothetic efficiency and shows how extended recoverability is still possible when HARP is violated but homothetic efficiency is high. Section 4 uses two data sets to apply the proposed method. Section 5 concludes. The appendix in Section A contains all proofs. A

\footnotetext{
${ }^{2}$ Silva and Stefanou (1996) provided a generalisation of these tests.
} 
supplementary "computable document" prepared with Wolfram Mathematica ${ }^{\circledR}$ that allows a graphical analysis of revealed preferred and worse sets recovered with our method can be downloaded from the internet (see Section 4.2).

\section{Preliminaries}

\subsection{Notation and Utility Maximisation}

The commodity space is $\mathbb{R}_{+}^{L}$ and the price space is $\mathbb{R}_{++}^{L}$, where $L \geq 2$ is the number of different commodities. ${ }^{3} \mathrm{~A}$ (competitive) budget set is defined as $B^{i}=B\left(\mathbf{p}^{i}\right)=$ $\left\{\mathbf{x} \in \mathbb{R}_{+}^{L}: \mathbf{p}^{i} \mathbf{x}^{i} \leq 1\right\}$, where $\mathbf{p}^{i}=\left(p_{1}^{i}, \ldots, p_{L}^{i}\right)^{\prime} \in \mathbb{R}_{++}^{L}$ is the price vector and wealth is normalised to $1 .{ }^{4}$ A demand function $D: \mathbb{R}_{++}^{L} \times \mathbb{R}_{++} \rightarrow \mathbb{R}_{+}^{L}$ of a consumer assigns to each budget set the commodity bundle chosen by the consumer. Unless otherwise noted, we assume that demand satisfies budget balancedness (i.e., $\mathbf{p}^{i} \mathbf{x}^{i}=1$ ). We assume that the only observables of the model are $N \geq 1$ different budgets and the corresponding demand of a consumer. The entire set of $N$ observations on a consumer is denoted as $\Omega=\left\{\left(\mathbf{x}^{i}, \mathbf{p}^{i}\right)\right\}_{i=1}^{N}$.

The bundle $\mathbf{x}^{i}$ is directly revealed preferred to a bundle $\mathbf{x}$, written $\mathbf{x}^{i} \mathrm{R}^{0} \mathbf{x}$, if $\mathbf{p}^{i} \mathbf{x}^{i} \geq \mathbf{p}^{i} \mathbf{x}$; it is strictly directly revealed preferred to $\mathbf{x}$, written $\mathbf{x}^{i} \mathrm{P}^{0} \mathbf{x}$, if $\mathbf{p}^{i} \mathbf{x}^{i}>\mathbf{p}^{i} \mathbf{x}$; it is revealed preferred to $\mathbf{x}$, written $\mathbf{x}^{i} \mathrm{R} \mathbf{x}$, if $\mathrm{R}$ is the transitive closure of $\mathrm{R}^{0}$, that is, if there exists a sequence $\mathrm{x}^{j}, \ldots, \mathrm{x}^{k}$, such that $\mathrm{x}^{i} \mathrm{R}^{0} \mathrm{x}^{j} \mathrm{R}^{0} \ldots \mathrm{x}^{k} \mathrm{R}^{0} \mathbf{x}$. The bundle $\mathbf{x}^{i}$ is strictly revealed preferred to $\mathbf{x}$, written $\mathrm{x}^{i} \mathrm{P} \mathbf{x}$, if $\mathbf{x}^{i} \mathrm{R} \mathbf{x}^{j} \mathrm{P}^{0} \mathbf{x}^{k} \mathrm{R} \mathbf{x}$ for some $j, k=1, \ldots, N$.

Definition 1 (Varian 1982) A set of observations $\Omega$ satisfies the Generalised Axiom of Revealed Preference (GARP) if for all $i, j=1, \ldots, N$, it holds that $\left[\right.$ not $\left.\mathbf{x}^{i} \mathrm{P}^{0} \mathbf{x}^{j}\right]$ whenever $\mathbf{x}^{j} \mathrm{R} \mathbf{x}^{i}$.

We say that a utility function $u: \mathbb{R}_{+}^{L} \rightarrow \mathbb{R}$ rationalises a set of observations $\Omega$ if $u\left(\mathbf{x}^{i}\right) \geq u(\mathbf{y})$ whenever $\mathbf{x}^{i} \mathrm{R}^{0} \mathbf{y}$. Let $\mathcal{U}$ denote the set of all continuous, non-satiated, monotonic, and concave utility functions. GARP is easily testable and a necessary and sufficient condition for utility maximisation, as Theorem 1 (Afriat's Theorem) below shows.

Theorem 1 (Afriat 1967, Diewert 1973, Varian 1982) The following conditions are equivalent:

\footnotetext{
${ }^{3}$ We use the following notation: For all $\mathbf{x}, \mathbf{y} \in \mathbb{R}^{L}, \mathbf{x} \geqq \mathbf{y}$ if $x_{i} \geq y_{i}$ for all $i=1, \ldots, L ; \mathbf{x} \geq \mathbf{y}$ if $\mathbf{x} \geqq \mathbf{y}$ and $\mathbf{x} \neq \mathbf{y} ; \mathbf{x}>\mathbf{y}$ if $x_{i}>y_{i}$ for all $i=1, \ldots, L$. We denote $\mathbb{R}_{+}^{L}=\left\{\mathbf{x} \in \mathbb{R}^{L}: \mathbf{x} \geqq(0, \ldots, 0)\right\}$ and $\mathbb{R}_{++}^{L}=\left\{\mathbf{x} \in \mathbb{R}^{L}: \mathbf{x}>(0, \ldots, 0)\right\}$.

${ }^{4}$ This normalisation is routinely applied in revealed preference analysis. The implicit assumption is that demand is homogeneous.
} 
1. the set of observations $\Omega$ satisfies GARP;

2. there exist numbers $U^{i}, \lambda^{i}>0$ such that

$$
U^{i} \leq U^{j}+\lambda^{j} \mathbf{p}^{j}\left(\mathbf{x}^{i}-\mathbf{x}^{j}\right)
$$

for all $i, j=1, \ldots, N$;

3. there exist numbers $V^{i}$ such that

$$
\begin{aligned}
& V^{i} \geq V^{j} \text { whenever } \mathbf{p}^{i} \mathbf{x}^{i} \geq \mathbf{p}^{i} \mathbf{x}^{j}, \text { and } \\
& V^{i}>V^{j} \text { whenever } \mathbf{p}^{i} \mathbf{x}^{i}>\mathbf{p}^{i} \mathbf{x}^{j}
\end{aligned}
$$

for all $i, j=1, \ldots, N$;

4. there exists a $u \in \mathcal{U}$ which rationalises the set of observations $\Omega$.

While conditions 1 and 2 can be found in Varian (1982), condition 3 is rather new in the literature. The intuition behind the inequalities in condition 3 is simple: If a consumer chooses the bundle $\mathbf{x}^{i}$ at prices $\mathbf{p}^{i}$ when $\mathbf{x}^{j}$ also was affordable, then he gains more utility from consuming $\mathbf{x}^{i}$, which is reflected by $V^{i}>V^{j}$ where $V^{i}$ can be thought of utility indices at time $i=1, \ldots, N$. Cherchye et al. (2015) use this condition to derive new non-parametric tests for weak separability based on solving mixed-integer linear programming problems. We will use similar ideas to formulate new methods to calculate efficiency indices.

\subsection{Utility Maximisation and Efficiency}

When a set of observations does not satisfy GARP, it is interesting to obtain a measure of how severe the violation is. One of the most popular measures for the severity of a violation is the Afriat Efficiency Index (AEI) due to Afriat (1972), also called the critical cost efficiency index (CCEI). ${ }^{5}$ Define, for some $e \in[0,1]$, the relation $\mathrm{R}^{0}(e)$ as $\mathbf{x}^{i} \mathrm{R}^{0}(e) \mathbf{x}^{j}$ if $e \mathbf{p}^{i} \mathbf{x}^{i} \geq \mathbf{p}^{i} \mathbf{x}$, and let $\mathrm{R}(e)$ be the transitive closure of $\mathrm{R}^{0}(e)$; furthermore, define the relation $\mathrm{P}^{0}(e)$ as $e \mathbf{p}^{i} \mathbf{x}^{i}>\mathbf{p}^{i} \mathbf{x}$. With these concepts, we can define a new version of GARP, called $\operatorname{GARP}(e)$.

\footnotetext{
${ }^{5}$ Reporting the AEI has become a standard for empirical studies, in particular experimental ones. See, for example, Sippel (1997), Mattei (2000), Harbaugh et al. (2001), Andreoni and Miller (2002), Février and Visser (2004), Choi et al. (2007b), Fisman et al. (2007), Dickinson (2009), Camille et al. (2011). See Gross (1995) for a survey of other measures. A common alternative to the AEI is the Houtman-Maks-Index (Houtman and Maks 1985) which is based on the maximal subset of a set of choices consistent with GARP; see also Heufer and Hjertstrand (2015) for a computationally feasibale approach to compute this index. Echenique et al. (2011) provided a new measure based on a money pump argument, and Dean and Martin (2015) provided a new measure based on the minimum cost of breaking all cycles or money pumps. Apesteguia and Ballester (2015) provided a measure based on welfare loss.
} 
Definition 2 A set of observations $\Omega$ satisfies $\operatorname{GARP}(e)$ for some $e \in(0,1]$ if for all $i, j=1, \ldots, N$, it holds that $\left[\right.$ not $\left.\mathbf{x}^{i} \mathrm{P}^{0}(e) \mathbf{x}^{j}\right]$ whenever $\mathbf{x}^{j} \mathrm{R}(e) \mathbf{x}^{i}$.

The AEI is the greatest number $e$ such that $\operatorname{GARP}(e)$ is satisfied; it is a measure of wasted income: If a consumer has an AEI of $e<1$, then he could have obtained the same level of utility by spending only a fraction of $e$ of what he actually spent to obtain this level. To compute the AEI when GARP is violated, Varian (1990) describes a binary search routine which he attributes to Houtman and Maks (1987).

The AEI is a summary statistic but does not provide information about which observed choices are causing the deviation from GARP. To get such information, we first define a further generalisation of $\operatorname{GARP}(e)$ :

Definition $3 A$ set of observations $\Omega$ satisfies $\operatorname{GARP}(\mathbf{v})$ for some $\mathbf{v} \in(0,1]^{N}$ if for all $i, j=1, \ldots, N$, it holds that $\left[\right.$ not $\left.\mathbf{x}^{i} \mathrm{P}^{0}\left(v_{i}\right) \mathbf{x}^{j}\right]$ whenever $\mathbf{x}^{j} \mathrm{R}\left(v_{j}\right) \mathbf{x}^{i}$.

Such a vector $\mathbf{v}$ can be a more disaggrated measure of efficiency. Varian (1993) defines one such measure, the violation index $\overline{\mathbf{v}}=\left(v_{1}, \ldots, v_{N}\right)$ with $\bar{v}_{i}=$ $\min _{\left\{j: \mathbf{x}^{j} \mathrm{R} \mathbf{x}^{i}\right\}} \mathbf{p}^{i} \mathbf{x}^{j}$. If the data satisfy GARP, then $\bar{v}_{i}=1$ for all $i$. Otherwise, $\bar{v}_{i}<1$ for some $i$, and this provides information about which $\mathbf{x}^{i}$ are problematic. Varian (1993) then proves the following proposition.

Proposition 1 (Varian 1993) A set of observations $\Omega$ satisfies $\operatorname{GARP}(\overline{\mathbf{v}})$.

Varian (1993) also notes that the vector $\overline{\mathbf{v}}$ does not, in general, give the minimum perturbation of budgets required. He provides an improved violation index which is computed using an iterative algorithm that determines the minimal $v_{i}$ for each $i$ required to break each revealed preference cycle in the data. See also Cox (1997) for a discussion of the improved violation index.

The 'exact' efficiency indices can be computed from the inequalities in Eqs. (2) and (3) using a simple mixed integer programming problem. To formulate this procedure, we initially make use of the fact that $\operatorname{GARP}(\mathbf{v})$ is equivalent to the following inequalities:

$$
\begin{aligned}
& V^{i} \geq V^{j} \text { whenever } v_{i} \mathbf{p}^{i} \mathbf{x}^{i} \geq \mathbf{p}^{i} \mathbf{x}^{j}, \text { and } \\
& V^{i}>V^{j} \text { whenever } v_{i} \mathbf{p}^{i} \mathbf{x}^{i}>\mathbf{p}^{i} \mathbf{x}^{j} .
\end{aligned}
$$

These inequalities are linear, and therefore suitable for empirical applications. Since there are unknowns entering both the left-hand and right-hand sides, we suggest using binary variables to link the two sides. ${ }^{6}$ Specifically, the inequalities in Eqs.

\footnotetext{
${ }^{6}$ See Cherchye et al. (2015) for a similar approach in the context of testing for weak separability of the utility function.
} 
(4) and (5) are equivalent to that there exist numbers $V^{i}$ and $X^{i j}$ such that, for all observations $i, j=1, \ldots, N$,

$$
\begin{aligned}
& V^{i}-V^{j}<X^{i j}, \\
&\left(X^{i j}-1\right) \leq V^{i}-V^{j}, \\
& v_{i} \mathbf{p}^{i} \mathbf{x}^{i}-\mathbf{p}^{i} \mathbf{x}^{j}<X^{i j} A^{i}, \\
&\left(X^{i j}-1\right) A^{j} \leq \mathbf{p}^{j} \mathbf{x}^{i}-v_{j} \mathbf{p}^{j} \mathbf{x}^{j}, \\
& 0 \leq V^{i}<1, \\
& X^{i j} \in\{0,1\},
\end{aligned}
$$

where $A^{i}>\mathbf{p}^{i} \mathbf{x}^{i}$ is a fixed number. We suggest to calculate the efficiency indices $\mathbf{v}$ by solving the following mixed integer linear programming (MiLP) problem ${ }^{7}$ with respect to $V^{i}, X^{i j}$ and $v_{i}$ :

$$
\min \sum_{i=1}^{N}\left(1-v_{i}\right) \text { subject to (c.i)-(c.vi) and } \mathbf{v} \in(0,1]^{N}
$$

Since any solution to a MiLP problem is a global solution, this problem is guaranteed to find a global optimum (in the L1-norm) in the efficiency indices v. Given this approach, we can formally define a vector efficiency index: We say that a vector $\tilde{\mathbf{v}}$ is a Varian Efficiency Vector (VEV) for $\Omega$ if $\Omega$ satisfies $\operatorname{GARP}(\tilde{\mathbf{v}})$ and there does not exist a $\mathbf{v}^{\prime} \geq \tilde{\mathbf{v}}$ such that $\Omega$ satisfies $\operatorname{GARP}\left(\mathbf{v}^{\prime}\right)$. When $\mathbf{v}$ is computed using the above MILP-approach, it will be a VEV.

\subsection{Recoverability of Preferences}

Preferences implicit in a set of data can be recovered with Varian's (1982) method: For some bundle $\mathbf{x}^{0} \in \mathbb{R}_{+}^{L}$ which was not necessarily observed as a choice, the set of prices which support $\mathbf{x}^{0}$ is defined as

$$
S\left(\mathbf{x}^{0}\right)=\left\{p^{0} \in \mathbb{R}_{++}^{L}:\left\{\left(\mathbf{x}^{i}, \mathbf{p}^{i}\right)\right\}_{i=0}^{N} \text { satisfies GARP and } \mathbf{p}^{0} \mathbf{x}^{0}=1\right\} .
$$

Varian (1982) uses $S\left(\mathbf{x}^{0}\right)$ to describe the set of all bundles which are revealed worse and revealed preferred to a bundle $\mathbf{x}^{0}$ : The set of all bundles which are revealed worse than $\mathbf{x}^{0}$ is given by

$$
R W\left(\mathbf{x}^{0}\right)=\left\{\mathbf{x} \in \mathbb{R}_{+}^{L}: \text { for all } \mathbf{p}^{0} \in S\left(\mathbf{x}^{0}\right), \mathbf{x}^{0} \mathrm{P} \mathbf{x}\right\}
$$

\footnotetext{
${ }^{7}$ Cherchye et al. (2008) have introduced mixed integer programming to the revealed preference literature.
} 
and the set of all bundles which are revealed preferred to $\mathbf{x}^{0}$ is given by

$$
R P\left(\mathbf{x}^{0}\right)=\left\{\mathbf{x} \in \mathbb{R}_{+}^{L}: \text { for all } \mathbf{p} \in S(\mathbf{x}), \mathbf{x} \mathrm{P}^{0}\right\} .
$$

The following fact follows directly from the definition; see also Varian (1982, Fact $3)$.

Fact $1 \mathbf{x} \in R W\left(\mathbf{x}^{0}\right)$ if and only if $\mathbf{x}^{0} \in R P(\mathbf{x})$.

The convex monotonic hull $C M H$ of a set of points $Y=\left\{\mathbf{y}^{i}\right\}_{i=1}^{M}$ is

$$
C M H(Y)=\text { convex hull of }\left(\left\{\mathbf{x} \in \mathbb{R}_{+}^{L}: \mathbf{x} \geq \mathbf{y}^{i} \text { for some } i=1, \ldots, M\right\}\right) .
$$

Let int $C M H(Y)$ denote the interior of $C M H(Y)$. The following Proposition provides an easy way of determining whether $\mathbf{x} \in R P\left(\mathbf{x}^{0}\right)$ and, by Fact 1 , also whether $\mathbf{x} \in R W\left(\mathbf{x}^{0}\right)$ (see Varian (1982) and Knoblauch (1992) for a proof).

Proposition 2 Suppose $\Omega$ satisfies GaRP. Then

$$
\operatorname{int} C M H\left(\left\{\mathbf{x}^{i}: \mathbf{x}^{i} \mathrm{R} \mathbf{x}^{0}\right\}\right) \subseteq R P\left(\mathbf{x}^{0}\right) \subseteq C M H\left(\left\{\mathbf{x}^{i}: \mathbf{x}^{i} \mathrm{R} \mathbf{x}^{0}\right\}\right) .
$$

Finally, note that variations of the sets $R P$ and $R W$ can still be constructed if GARP is violated. Obviously, $S\left(\mathbf{x}^{0}\right)$ will be empty in this case, but based on Proposition 2, one can still compute the convex monotonic hulls and analyse the result. However, this will necessarily lead to intersection of $R P(\mathbf{x})$ and $R W(\mathbf{x})$ for some $\mathbf{x}$. Thus, it would more appropriate to base the constructions on $\mathrm{R}(e)$ or $\mathrm{R}(\mathbf{v})$, and to define $S\left(\mathbf{x}^{0}\right)$ in terms of $\operatorname{GaRP}(e)$ or $\operatorname{GARP}(\mathbf{v})$.

\subsection{Homotheticity}

\subsubsection{Definition and Tests}

Homotheticity is a restriction on preferences. We say that a utility function is homothetic if it is a positive monotonic transformation of a linearly homogeneous utility function; that is, if $u(\mathbf{x})>u(\mathbf{y})$ then $u(\lambda \mathbf{x})>u(\lambda \mathbf{y})$ for all $\lambda>0$. Varian (1983) provides the following axiom, which he shows is equivalent to homothetic rationalisation (Theorem 2 ).

Definition 4 (Varian 1983) A set of observations $\Omega$ satisfies the Homothetic Axiom of Revealed Preference (HARP) if for all distinct choices of indices $i, j, k, \ldots, \ell$, it holds that $\left(\mathbf{p}^{i} \mathbf{x}^{j}\right)\left(\mathbf{p}^{j} \mathbf{x}^{k}\right) \cdots\left(\mathbf{p}^{\ell} \mathbf{x}^{i}\right) \geq 1$. 
Theorem 2 (Varian 1983) The following conditions are equivalent:

1. the set of observations $\Omega$ satisfies HARP;

2. there exist numbers $U^{i}>0$ such that

$$
U^{i} \leq U^{j} \mathbf{p}^{j} \mathbf{x}^{i}
$$

for $i, j=1, \ldots, N$;

3. there exists a homothetic $u \in \mathcal{U}$ which rationalises the set of observations $\Omega$.

\subsubsection{Homothetic Recoverability of Preferences}

Following Knoblauch (1993), define for a set of observations which satisfies HARP a scalar

$$
t_{i, \diamond}=\min \left\{\left(\mathbf{p}^{i} \mathbf{x}^{j}\right)\left(\mathbf{p}^{j} \mathbf{x}^{k}\right) \cdots\left(\mathbf{p}^{\ell} \mathbf{x}^{\diamond}\right)\right\},
$$

where the minimum is over all finite sequences $i, j, \ldots, \ell$ between 1 and $N$ inclusive, and $t_{\diamond, \diamond}=1$. Note that $\diamond$ can be the index of an observed choice $\mathbf{x}^{m}$ or be equal to 0 for a bundle $\mathbf{x}^{0}$ which was not observed as a choice. We can compute $t_{i, 0}$ for any arbitrary bundle $\mathbf{x}^{0}$ as we do not need a price vector $\mathbf{p}^{0}$. We say that $t_{i, \diamond} \mathbf{x}^{i}$ is homothetically revealed preferred to $\mathbf{x}^{\diamond}$, written $t_{i, \diamond} \mathbf{x}^{i} \mathrm{H} \mathbf{x}^{\diamond}$. The scalar $t=t_{i, \diamond}$ is the smallest value such that $t \mathbf{x}^{i} H \mathbf{x}^{\diamond}$. Note that if $t_{i, \diamond}=\left(\mathbf{p}^{i} \mathbf{x}^{j}\right) \cdots\left(\mathbf{p}^{k} \mathbf{x}^{\ell}\right) \cdots\left(\mathbf{p}^{m} \mathbf{x}^{\diamond}\right)$, then $t_{i, \diamond}=t_{i, k} t_{\ell, \diamond}$.

Figure 1 illustrates the scalar factors in Eq. (12) with an example with three observations. In (a), we see that $t_{2,3}=\mathbf{p}^{2} \mathbf{x}^{3}$. In (b), $\mathbf{x}^{1}$ can be scaled up so that it still is homothetically revealed preferred to $t_{2,3} \mathbf{x}^{2}$, and we find that $t_{1,3}=$ $\left(\mathbf{p}^{1} \mathbf{x}^{2}\right)\left(\mathbf{p}^{2} \mathbf{x}^{3}\right)=t_{1,2} t_{2,3}$. This is not a coincidence, as in two dimensions budgets can be sorted by their price ratio. If budgets are sorted and $B^{1}$ and $B^{N}$ have the lowest and highest price ratio, respectively, then $t_{1, N}=\left(\mathbf{p}^{1} \mathbf{x}^{2}\right)\left(\mathbf{p}^{2} \mathbf{x}^{3}\right) \cdots\left(\mathbf{p}^{N-1} \mathbf{x}^{N}\right)$, as was shown in Heufer (2013).

Knoblauch (1993) also shows how to recover homothetic preferences implicit in a set of observations which satisfies HARP. Define the set of bundles which are homothetically revealed preferred to $\mathbf{x}^{\diamond}$ as

$$
H R P\left(\mathbf{x}^{\diamond}\right)=\operatorname{int} C M H\left(\mathbf{x}^{\diamond} \cup \bigcup_{i=0}^{N} t_{i, \diamond} \mathbf{x}^{i}\right)
$$

The set $H R P\left(\mathbf{x}^{\diamond}\right)$ is very useful indeed, as Theorem 3 below shows that it describes the set of bundles which any rationalising homothetic utility function must rank higher than $\mathbf{x}^{0}$. Define the set of bundles which are homothetically revealed worse to $\mathbf{x}^{\diamond}$ as

$$
H R W\left(\mathbf{x}^{\diamond}\right)=\left\{\mathbf{x} \in \mathbb{R}_{+}^{L}: \mathbf{x}^{\diamond} \in H R P(\mathbf{x})\right\} .
$$




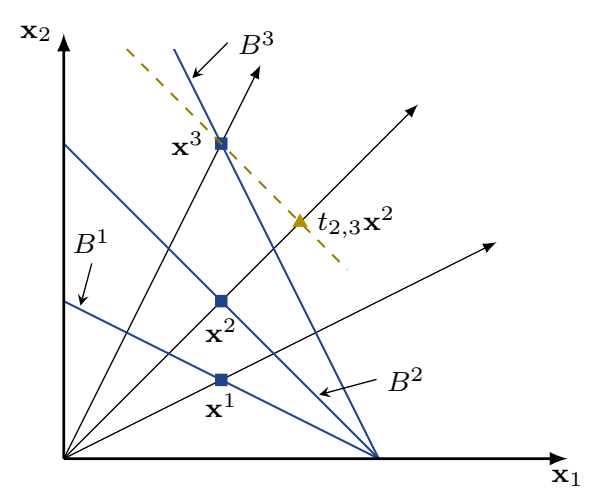

(a)

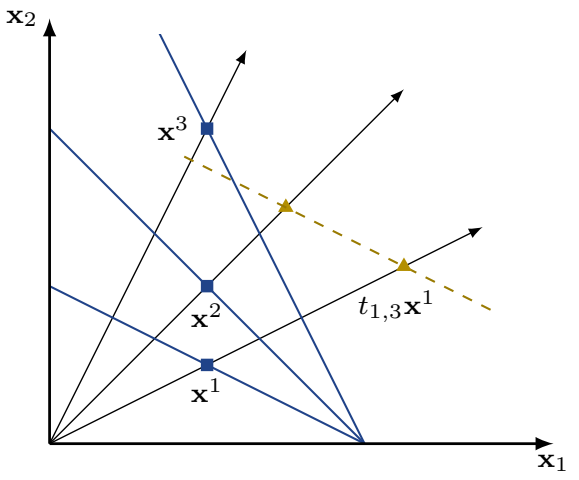

(b)

Figure 1: Illustration of the scalar factors.

As $H R P$ can be easily computed as the convex hull of a finite number of points, it is also easy to test for any bundle if $\mathbf{x} \in H R W\left(\mathbf{x}^{\diamond}\right)$.

Theorem 3 (Knoblauch 1993) Suppose $\Omega$ satisfies HARP. The following conditions are equivalent:

1. $\mathbf{x} \in \operatorname{HRP}\left(\mathbf{x}^{\diamond}\right)$;

2. every homothetic $u \in \mathcal{U}$ which rationalises $\Omega$ satisfies $u(\mathbf{x})>u\left(\mathbf{x}^{\diamond}\right)$.

See Knoblauch (1993) for the proof. The following corollary is then straightforward, and we omit the proof.

Corollary 1 Suppose $\Omega$ satisfies HARP. The following conditions are equivalent: 1. $\mathbf{x} \in H R W\left(\mathbf{x}^{\diamond}\right)$;

2. every homothetic $u \in \mathcal{U}$ which rationalises $\Omega$ satisfies $u\left(\mathbf{x}^{\diamond}\right)>u(\mathbf{x})$.

It should also be obvious that $R P\left(\mathbf{x}^{\diamond}\right) \subseteq H R P\left(\mathbf{x}^{\diamond}\right)$ and $R W\left(\mathbf{x}^{\diamond}\right) \subseteq H R W\left(\mathbf{x}^{\diamond}\right)$ for all $\mathbf{x}^{\diamond}$.

\section{Homothetic Efficiency}

\subsection{A Lower Bound on Homothetic Efficiency}

Suppose we have a set $\Omega=\left\{\left(\mathbf{x}^{i}, \mathbf{p}^{i}\right)\right\}_{i=1}^{2}$. If the consumer had homothetic preferences, then his demand when facing budget $B\left(\mathbf{p}^{1} / t\right)$ would be $t \mathbf{x}^{1}$. Then the smallest $t$ for which $t \mathbf{x}^{1}$ would be revealed preferred to $\mathbf{x}^{2}$ is $t=\mathbf{p}^{1} \mathbf{x}^{2}$. Now suppose $\Omega$ does not satisfy HARP such that $\left(\mathbf{p}^{1} \mathbf{x}^{2}\right)\left(\mathbf{p}^{2} \mathbf{x}^{1}\right)<1$. Then the choice $\left(\mathbf{p}^{1} \mathbf{x}^{2}\right) \mathbf{x}^{1}$ on $B\left(\mathbf{p}^{1} / \mathbf{p}^{1} \mathbf{x}^{2}\right)$ would be revealed preferred to $\mathbf{x}^{2}$, but as HARP is violated, $\mathbf{p}^{2} \mathbf{x}^{2}=1>\left[\mathbf{p}^{2}\left(\mathbf{p}^{1} \mathbf{x}^{2}\right) \mathbf{x}^{1}\right]=\left(\mathbf{p}^{1} \mathbf{x}^{2}\right)\left(\mathbf{p}^{2} \mathbf{x}^{1}\right)$, that is, $\mathbf{x}^{2}$ is strictly revealed preferred to 
$\left(\mathbf{p}^{1} \mathbf{x}^{2}\right) \mathbf{x}^{1}$, which would violate GARP. But if we relax GARP, as is done to compute the AEI, we can find the greatest $e \in(0,1]$ such that $e \mathbf{p}^{2} \mathbf{x}^{2}=e \leq\left[\mathbf{p}^{2}\left(\mathbf{p}^{1} \mathbf{x}^{2}\right) \mathbf{x}^{1}\right]$, that is, $e=\left(\mathbf{p}^{1} \mathbf{x}^{2}\right)\left(\mathbf{p}^{2} \mathbf{x}^{1}\right)$. This $e$ has a similar economic interpretation as the AEI: If the preferences of the consumer were homothetic but demand is specified with errors, then $e$ can be interpreted as a level of expenditure which the consumer wasted due to the errors; in particular, he could have obtained the same utility as he obtained from choosing $\mathbf{x}^{2}$ at an expenditure $e<1$ rather than the actual expenditure of 1 .

However, if we use this approach, the multiplication of the scalars $\mathbf{p}^{i} \mathbf{x}^{j}$ can lead to very low values of $e$. To illustrate the general problem with a hypothetical scenario, suppose that a subject is asked to make ten choices from the same budget set. Suppose we observe $\mathbf{x}^{i}=\mathbf{x}^{j}$ for all $i, j=1, \ldots, N$, with $\mathbf{p}^{i}=\mathbf{p}^{j}$ for all $i, j=1, \ldots, N$ as well. Suppose that $\mathbf{p}^{i} \mathbf{x}^{i}=.95$ (i.e., budget balancedness is violated). Then $\left(\mathbf{p}^{1} \mathbf{x}^{2}\right)\left(\mathbf{p}^{2} \mathbf{x}^{3}\right) \cdots\left(\mathbf{p}^{10} \mathbf{x}^{1}\right) \approx 0.5987$, even though only five percent of the wealth level was wasted each time and even though the data would satisfy HARP if the $w^{i}$ were set to .95. But even without violations of budget balancedness and with different budgets, minor errors can lead to very low values of $e$ if many choices are observed.

We therefore suggest to use the following axiom, called $\operatorname{HARP}(e)$, which takes into account the number of scalars which are multiplied.

Definition $5 A$ set of observations $\Omega$ satisfies $\operatorname{HARP}(e)$ for some $e \in(0,1]$ if for all distinct choices of indices $i, j, k, \ldots, \ell$, it holds that

$$
\left(\frac{\mathbf{p}^{i} \mathbf{x}^{j}}{e}\right)\left(\frac{\mathbf{p}^{j} \mathbf{x}^{k}}{e}\right) \cdots\left(\frac{\mathbf{p}^{\ell} \mathbf{x}^{i}}{e}\right) \geq 1 .
$$

Figure 2 illustrates the idea. Figure 2.(a) shows the two observations. The dashed line shows the boundary of the shifted budget $B^{1}$ which contains $\mathbf{x}^{2}$. The intersection of the dashed line and the ray through the origin and $\mathbf{x}^{1}$, shown as $\lambda \mathbf{x}^{1}$, gives the demand on the shifted budget if preferences were homothetic. Here $\lambda$ is chosen to equal $\mathbf{p}^{1} \mathbf{x}^{2}$; note that, by Theorem $3, \lambda \mathbf{x}^{1}$ would be homothetically revealed preferred to $\mathbf{x}^{2}$ if preferences were homothetic. But as $\lambda \mathbf{x}^{1}$ is in the interior of budget $B^{2}, \mathbf{x}^{2}$ is strictly revealed preferred to $\lambda \mathbf{x}^{1}$, thus HARP is violated. Also note that there is a $\mu<1$ such that $\mu \mathbf{x}^{2}$ would be homothetically revealed preferred to $\lambda \mathbf{x}^{1}$. Then $\tilde{\lambda} \mathbf{x}^{1}$ with $\tilde{\lambda}<\lambda$ would be homothetically revealed preferred to $\mu \mathbf{x}^{2}$. This process can be repeated ad infinitum.

Figure 2.(b) shows the two budgets shifted downwards by setting the wealth level to $e<1$. Figure 2.(c) shows that if $\mathbf{x}^{1}$ is scaled upwards by a factor equal to $\lambda / e$, we find that while $\mathbf{x}^{2}$ is still strictly revealed preferred to it $-\mathbf{x}^{2} \mathrm{P}^{0}\left(\lambda \mathbf{x}^{1} / e\right)$ - it is not strictly revealed preferred at efficiency level $e-\left[\right.$ not $\left.\mathrm{x}^{2} \mathrm{P}^{0}(e)\left(\lambda \mathbf{x}^{1} / e\right)\right]$. This is indeed the smallest $e$ for which $\operatorname{HARP}(e)$ is satisfied; as the example has 
only two observations, this $e$ can be found by setting $\left[\left(\mathbf{p}^{1} \mathbf{x}^{2}\right) / e\right]\left[\left(\mathbf{p}^{2} \mathbf{x}^{1}\right) / e\right]=1$ and solving for $e$.

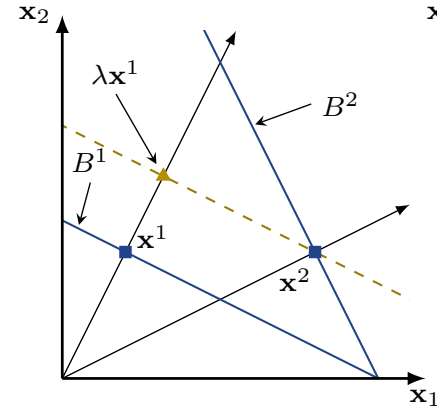

(a)

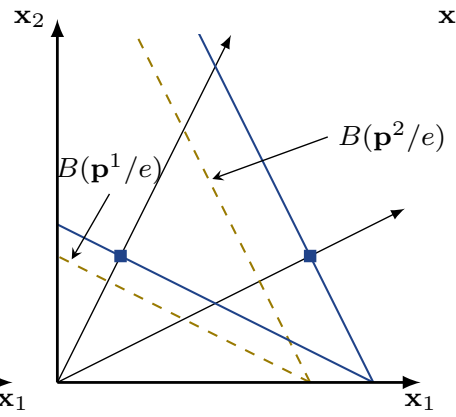

(b)

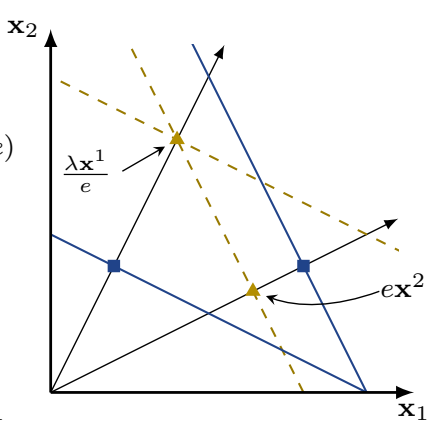

(c)

Figure 2: An illustration of $\operatorname{HARP}(e)$ and the homothetic efficiency index. The example uses $\mathbf{x}^{1}=(2,4), \mathbf{p}^{1}=(1 / 10,1 / 5), \mathbf{x}^{2}=(8,4)$, and $\mathbf{p}^{2}=(1 / 10,1 / 20)$. The greatest $e$ for which $\operatorname{HARP}(e)$ is satisfied is $4 / 5$.

Given HARP(e), we propose the following definition, in analogy to the AEI:

Definition 6 For a set of observations $\Omega$, the Homothetic Efficiency Index (HEI) is the greatest $e \in(0,1]$ such that $\Omega$ satisfies $\operatorname{HARP}(e)$.

Note that the HEI cannot increase as the number of observations increase; it can only decrease as more observations are added. This is because the HEI is the minimum over all simple cycles in the data (such as going from $\mathbf{x}^{i}$ to itself in the definition of the axiom $\operatorname{HARP}(e))$. The cycle that determined the old minimum remains in the data as more observations are added. ${ }^{8}$

The HeI can be computed with the same binary search algorithm used for calculating the AEI (simply exchange $\operatorname{GARP}(e)$ for $\operatorname{HARP}(e)$ in the algorithm). It can be reported as a summary statistic. The next theorem will provide a good motivation and justification to compute and report $e$. It is based on a concept we call $e$-rationalisation, which is in the same spirit as the definitions proposed and analysed by Halevy et al. (2012).

Definition 7 A utility function $u \in \mathcal{U}$ e-rationalises a set of observations $\Omega$ if $u\left(\mathbf{x}^{i}\right) \geq u(\mathbf{y})$ whenever $\mathbf{x}^{i} \mathrm{R}^{0}(e) \mathbf{y}$.

For $e$ close to 1, a utility function which $e$-rationalises a set of data still adequately explain choices as the result of utility maximisation with minor errors.

\footnotetext{
${ }^{8}$ Suppose there are only two observations, and HARP is violated. Then HEI $=\left[\left(\mathbf{p}^{1} \mathbf{x}^{2}\right)\left(\mathbf{p}^{2} \mathbf{x}^{1}\right)\right]^{1 / 2}$ If a third observation is introduced, then for example $\left[\left(\mathbf{p}^{1} \mathbf{x}^{3}\right)\left(\mathbf{p}^{3} \mathbf{x}^{2}\right)\left(\mathbf{p}^{2} \mathbf{x}^{1}\right)\right]^{1 / 3}$ could be less that the former HEI in which case efficiency decreases; but it cannot lead to an increase of the HEI as the cycle from observation 1 to itself via observation 2 is still in the data.
} 
The following theorem shows that $\operatorname{HARP}(e)$ is necessary and sufficient for homothetic e-rationalisation.

Theorem 4 For any $e \in[0,1]$ the following conditions are equivalent:

1. the set of observations $\Omega$ satisfies $\operatorname{HARP}(e)$;

2. there exist numbers $U^{i}>0$ such that

$$
e U^{i} \leq U^{j} \mathbf{p}^{j} \mathbf{x}^{i}
$$

for $i, j=1, \ldots, N$;

3. there exists a homothetic $u \in \mathcal{U}$ which e-rationalises the set of observations $\Omega$.

Theorem 4 also shows why we can still interpret the HEI as a measure of wasted income, just as we do with the AEI. Suppose a homothetic utility function $u$ $e$-rationalises $\Omega$, but $u(y)>u\left(x^{i}\right)$ even though $\mathbf{x}^{i} \mathrm{R} \mathbf{y}$. This contradiction of the ranking of bundles by $u$ with $\mathrm{R}$ disappears if we assume that the consumer wasted a fraction $e$ of his income, because by $e$-rationalisation we must have $\mathbf{x}^{i} \mathrm{R}(e) \mathbf{y}$.

\subsection{Improved Homothetic Efficiency Vector}

Similar to the case of the AEI and Varian's (1993) improved violation index, the HEI is only a lower bound on homothetic efficiency. A homothetic efficiency vector which provides information about how much each budget has to be perturbed to achieve a meaningful kind of consistency while keeping the perturbations minimal would be informative and useful for applied work. We suggest the following straightforward generalisation of $\operatorname{HARP}(e)$.

Definition 8 A set of observations $\Omega$ satisfies $\operatorname{HARP}(\mathbf{h})$ for some $\mathbf{h}=\left(h_{1}, \ldots, h_{N}\right) \in$ $(0,1]^{N}$ if for all $i, j=1, \ldots, N$, it holds that

$$
\left(\frac{\mathbf{p}^{i} \mathbf{x}^{j}}{h_{i}}\right)\left(\frac{\mathbf{p}^{j} \mathbf{x}^{k}}{h_{j}}\right) \cdots\left(\frac{\mathbf{p}^{\ell} \mathbf{x}^{i}}{h_{\ell}}\right) \geq 1 .
$$

The problem with computing a vector $\mathbf{h}$ with maximal values is that "breaking cycles" is not as easy as in the standard case in Varian (1993). It is not feasible to consider breaking "homothetically revealed preference cycles". If a set of data does not satisfy HARP, then Knoblauch's (1993) concept of "homothetically revealed preferred to" is either ill defined or computationally infeasible for many observations. If in the definition of the scalar factors in Eq. (12) we allow for multiple occurrences of indices, then the minimum is not defined and the infimum is 0 . For example, if $\left(\mathbf{p}^{1} \mathbf{x}^{2}\right)\left(\mathbf{p}^{2} \mathbf{x}^{1}\right)<1$, then $\lim _{n \rightarrow \infty}\left[\left(\mathbf{p}^{1} \mathbf{x}^{2}\right)\left(\mathbf{p}^{2} \mathbf{x}^{1}\right)\right]^{n}=0$. If we restrict Eq. (12) 
to distinct vertices, then the problem of computing the scalars amounts to the NP-hard problem of finding a simple shortest path in a weighted complete graph (i.e., a path that visits each vertex at most once, except for the first vertex if the path is a cycle). The complexity of this endeavour quickly approaches a level which makes computation infeasible. ${ }^{9}$

We will therefore rely on a (mixed-integer) linear programming approach. The basis for this approach will be provided by a theorem which is the analogue of Theorem 4 (the proof is practically the same as for Theorem 4 and we omit it).

Definition 9 A utility function $u \in \mathcal{U} \mathbf{h}$-rationalises a set of observations $\Omega$ if $u\left(\mathbf{x}^{i}\right) \geq u(\mathbf{y})$ whenever $\mathbf{x}^{i} \mathrm{R}^{0}\left(h_{i}\right) \mathbf{y}$.

Theorem 5 For any $\mathbf{h}=\left(h_{1}, \ldots, h_{N}\right) \in[0,1]^{N}$ the following conditions are equivalent:

1. the set of observations $\Omega$ satisfies $\operatorname{HARP}(\mathbf{h})$;

2. there exist numbers $U^{i}>0$ such that:

$$
h_{j} U^{i} \leq U^{j} \mathbf{p}^{j} \mathbf{x}^{i}
$$

for $i, j=1, \ldots, N$

3. there exists a homothetic $u \in \mathcal{U}$ which $\mathbf{h}$-rationalises the set of observations $\Omega$.

We say that $\tilde{\mathbf{h}}$ is a Homothetic Efficiency Vector (HEv) for $\Omega$ if $\Omega$ satisfies $\operatorname{HARP}(\tilde{\mathbf{h}})$ and there does not exist a $\mathbf{h}^{\prime} \geq \tilde{\mathbf{h}}$ such that $\Omega$ satisfies $\operatorname{HARP}\left(\mathbf{h}^{\prime}\right)$.

As explained above, calculating the HEv corresponds to solving a NP-hard problem. However, it is possible to compute a first order approximation of the Hev in polynomial time. To see how, we first define $\kappa_{i}=\log \left(h_{i}\right)$ for all $i=1, \ldots, N$ and note that a first order Taylor expansion of $\log \left(h_{i}\right)$ about the point 1 yields $\log \left(h_{i}\right) \simeq-\left(1-h_{i}\right)$, so that

$$
\begin{aligned}
\arg \max \sum_{i=1}^{N} \kappa_{i} & =\arg \max \sum_{i=1}^{N} \log \left(h_{i}\right) \\
& \simeq \arg \max \sum_{i=1}^{N}-\left(1-h_{i}\right)=\arg \min \sum_{i=1}^{N}\left(1-h_{i}\right) .
\end{aligned}
$$

\footnotetext{
${ }^{9}$ In a complete graph with $N \geq 2$ vertices, there are $\sum_{i=2}^{N} \frac{(N-2) !}{(N-i) !}$ different simple paths between two distinct vertices. For the 50 observations per subject collected in Fisman et al. (2007) and Choi et al. (2007a), this would require to compare $3.37445 \cdot 10^{61}$ different paths.
} 
Next to note is that the left hand side of the inequalities in Eq. (16) is positive, implying that they can be equivalently rewritten as (by a log-linearisation):

$$
\kappa_{j}+u^{i}-u^{j} \leq \log \left(\mathbf{p}^{j} \mathbf{x}^{i}\right),
$$

for all $i, j=1, \ldots, N$, where $u^{i}=\log \left(U^{i}\right)$ and $\kappa_{j}=\log \left(h_{j}\right)$. Consider the following linear programme (solved with respect to $\kappa_{i} \in(-\infty, 0]$ and $u^{i} \in(-\infty, \infty)$ for all $i=1, \ldots, N)$ :

$$
\max \sum_{i=1}^{N} \kappa_{i} \text { subject to (18), }
$$

and define the optimal solutions from this problem as $\hat{\kappa}_{i}$ for all $i=1, \ldots, N$. Given the Taylor approximation in (17), the set of numbers $\hat{h}_{i}=\exp \left(\hat{\kappa}_{i}\right)$ is then a first order approximation to the HEv (in the L1-norm).

There is an equivalent way to express the linear problem (19). This follows from that $\sum_{i=1}^{N} \log \left(h_{i}\right)$ is a monotonic log-transformation of $\prod_{i=1}^{N} h_{i}$, in which case (19) can be rephrased as:

$$
\max \prod_{i=1}^{N} h_{i} \text { subject to }(16),
$$

This reformulation shows that the problem (19) does not compute the set of indices closest to the unit vector in a 'true' norm. Despite this, computing the indices from (19) has several advantages over, for example, computing them closest to the unit vector in the Minkowski norm. ${ }^{10}$ First, it is a computationally tractable procedure for large data sets since it can be solved with elementary linear programming techniques, i.e., in polynomial time (which is not the case for any optimisation problem based on, for example, the Minkowski norm). Second, as our empirical applications show, the problem (19) seems to provide a very good approximation of the $\mathrm{HEV}$, and the approximation becomes more accurate the closer the $\mathrm{HEV}$ is to the unit vector. Third, rephrasing a potentially intractable problem based on a first order approximation is a commonly used procedure to find maximal elements in constrained optimisation problems.

As discussed in the introduction, our methods have a wide range of potential applications. In the next section, we apply them to experimental and survey

\footnotetext{
${ }^{10}$ Computing the set of indices closest to the unit vector in the Minkowski norm would amount to solving the problem: $\min \left(\sum_{i=1}^{N}\left(1-h_{i}\right)^{\varphi}\right)^{1 / \varphi}$ subject to (16) for some $\varphi \geq 1$. Computing the indices as close to 1 as possible in the L1-norm corresponds to solving this problem for $\varphi=1$.
} 
data. This kind of data often comprise panels of a large number of subjects (in controlled experiments) or households (in survey studies) which are observed over a limited number of time periods. For example, our experimental data set consists of choices of subjects over $T=50$ decision rounds while the survey data consists of household consumption allocations in up to $T=8$ consecutive time periods. Thus, the number of time observations encountered in experimental or survey data applications generally does not pose any problems in applying our methods. The panel dimension (i.e., the number of subjects or households) is usually not of any concern since each subject or household is analysed and treated individually. More precisely, the efficiency indices are calculated for every subject or household, which allow us to avoid (debatable) preference homogeneity assumptions across subjects or households. This approach effectively accounts for heterogeneity between subjects and optimally exploits the panel structure of the data.

Some microeconomic applications may consist of cross-sections with thousands of observations. ${ }^{11}$ One approach to working with these kinds of data is to split the full sample into sub samples based on observable demographic factors, and apply the proposed methods to the smaller sub samples. Although this does not allow to analyse "between" group heterogeneity, it still allows for analysing "within" group heterogeneity, which may be of more interest to the researcher.

\subsection{Recoverability with Improved Homothetic Efficiency}

Homothetic recoverability with data adjusted by efficiency indices is not as straightforward in the standard case described in Section 2.3. It is not sufficient to divide the scalar factors $t_{i, \diamond}$ in Eq. (12) by $h_{i}$, or to divide each component in Eq. (12) by $h_{i}$ to find new scalar factors. Figure 2.(c) already illustrates this: With only two observations, we would have $t_{1,2}=\mathbf{p}^{1} \mathbf{x}^{2}$ if the data satisfied HARP. But here, $\lambda=\mathbf{p}^{1} \mathbf{x}^{2}$, and clearly, $\mathbf{x}^{2}$ is still revealed preferred to $\lambda \mathbf{x}^{1} / e$. We need to scale down $\mathbf{x}^{1}$ by $e$ as well to remove this contradiction, so $\lambda \mathbf{x}^{1} / e$ is only homothetically revealed preferred to $e \mathbf{x}^{2}$.

Recoverability with adjustment by homothetic efficiency can be done for both the HEI and the HEv. Both approaches are very similar, but the HEv is superior, which is why we only present this case here. Let $\tilde{\mathbf{h}}$ be an HEv of a set of observations $\Omega$. Define

$$
\tilde{t}_{i, \diamond}=\min \left\{\left(\frac{\mathbf{p}^{i} \mathbf{x}^{j}}{h_{i}^{2}}\right)\left(\frac{\mathbf{p}^{j} \mathbf{x}^{k}}{h_{j}^{2}}\right) \cdots\left(\frac{\mathbf{p}^{\ell} \mathbf{x}^{\diamond}}{h_{\ell}^{2}}\right)\right\},
$$

\footnotetext{
${ }^{11}$ Importantly, our methods also meet the data requirements for other types of applications. For example, applications to macroeconomics and trade theory are often based on quarterly or monthly time series or panel data that spans in general up to five or six decades, for which our methods are computationally tractable.
} 
where the minimum is over all finite sequences $i, j, \ldots, \ell$ between 1 and $N$ inclusive. We say that $\tilde{t}_{i, \diamond} \mathbf{x}^{i}$ is homothetically revealed preferred at efficiency level $h_{i}$ to $\mathbf{x}^{\diamond}$, written $\tilde{t}_{i, \diamond} \mathbf{x}^{i} \tilde{\mathrm{H}} \mathbf{x}^{\diamond}$. To motivate this definition and show that it is useful, we first need to define the set of bundles which are homothetically revealed preferred at efficiency vector $\tilde{\mathbf{h}}$ to $\mathbf{x}^{\diamond}$ as

$$
\widetilde{H R P}_{\tilde{\mathbf{h}}}\left(\mathbf{x}^{\diamond}\right)=\operatorname{int} C M H\left(\mathbf{x}^{\diamond} \cup \bigcup_{i=1}^{N} \tilde{t}_{i, \diamond} \mathbf{x}^{i}\right)
$$

and

$$
\widetilde{H R W}_{\tilde{\mathbf{h}}}\left(\mathbf{x}^{\diamond}\right)=\left\{\mathbf{x} \in \mathbb{R}_{+}^{L}: \mathbf{x}^{\diamond} \in \widetilde{H R P}_{\tilde{\mathbf{h}}}(\mathbf{x})\right\}
$$

Figure 3 shows an example of the sets $\widetilde{H R P}_{\tilde{\mathbf{h}}}\left(\mathbf{x}^{0}\right)$ and $\widetilde{H R W}_{\tilde{\mathbf{h}}}\left(\mathbf{x}^{0}\right)$. Figure 4 in the next section shows four examples using experimental data by Fisman et al. (2007).

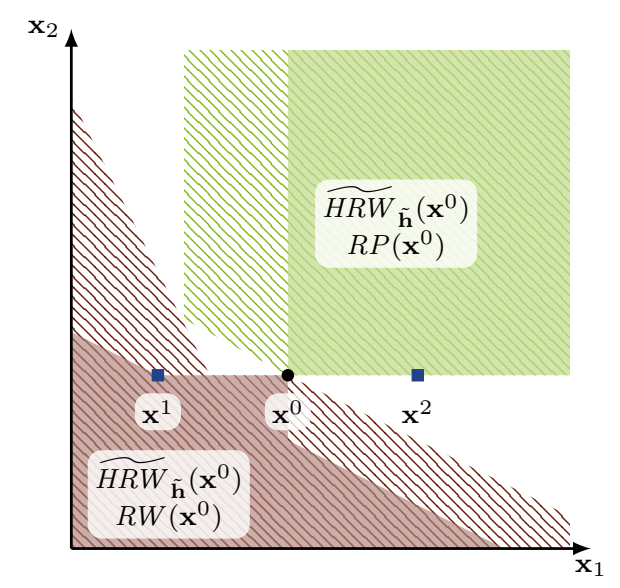

Figure 3: (Homothetically) revealed preferred and worse sets for $\mathrm{x}^{0}=\left(\mathrm{x}^{1}+\mathrm{x}^{2}\right) / 2$ with the same data as in Figure 2 based on a HEv with $\tilde{\mathbf{h}}=(1,16 / 25)$. The hatched area shows $\widehat{H R P}$ and $\widehat{H R W}$, the filled area $R P$ and $R W$.

While Theorem 4 provides the motivation to report the HEI as a summary statistic, and Theorem 5 motivates the Hev, Theorem 6 below justifies the use of $\widetilde{H R P}_{\tilde{\mathbf{h}}}$ and $\widetilde{H R W}_{\tilde{\mathbf{h}}}$. Note that it is not necessarily the case that $R P\left(x^{0}\right) \subseteq H R P_{\tilde{\mathbf{h}}}\left(x^{0}\right)$. This can happen if homothetic efficiency is particularly low. However, as the application in Section 4.2 demonstrates, this is not a problem for practical purposes when homothetic efficiency is not unreasonably low. Furthermore, Theorem 6 provides a strong justification: It shows that every homothetic utility function 
which $\mathbf{h}$-rationalises the data will agree with our construction of the homothetically revealed preferred and worse sets.

Theorem 6 If $\Omega$ satisfies $\operatorname{HARP}(\mathbf{h})$, then for every homothetic $u \in \mathcal{U}$ which h-rationalises $\Omega$,

$$
\begin{aligned}
& \widetilde{H R P}_{\mathbf{h}}\left(\mathbf{x}^{\diamond}\right) \subseteq\left\{\mathbf{x} \in \mathbb{R}_{+}^{L}: u(\mathbf{x}) \geq u\left(\mathbf{x}^{\diamond}\right)\right\} \\
& \widetilde{H R W}_{\mathbf{h}}\left(\mathbf{x}^{\diamond}\right) \subseteq\left\{\mathbf{x} \in \mathbb{R}_{+}^{L}: u(\mathbf{x}) \leq u\left(\mathbf{x}^{\diamond}\right)\right\}
\end{aligned}
$$

\section{Applications}

Our aim with this empirical exercise is threefold: First, we want to show that our methods can recover detailed information about subjects' preferences in experimental data sets. Second, we want to show that homothetic efficiency can be high for consumer choice data. Finally, we want to show that data which is adjusted by the HEV (HEI) can have much higher discriminatory power against random behaviour than data adjusted by the VEV (AEI).

\subsection{Test Power and Conditional Test Power}

The standard approach to calculate the power for revealed preference tests is based on Bronars (1987). In this paper, we follow Bronars' approach and generate many random choice sets uniformly distributed on the budget sets and compute the fraction of sets that either violates GARP or HARP, which we refer to as the power of GARP and HARP, respectively.

To analyse the loss in power for expenditure-adjusted data, we employ the following three-step procedure: (i) We compute the efficiency index using the observed data; (ii) Then, in a second step, we generate random data sets using Bronars' approach; (iii) Finally, we calculate the fraction of sets violating GARP or HARP, where total expenditure is adjusted for efficiency. More precisely, we deflate expenditure by the efficiency index computed in the first step when testing whether the randomly generated data sets satisfies GARP or HARP. Repeating the threestep procedure for all four efficiency indices AEI, VEv, HeI, and Hev allow us to compare the loss in discriminatory power across the indices.

A potential issue with calculating the power for HARP in Bronars' framework is that GARP is a necessary condition for HARP, and the HEI can never exceed the AEI. It would therefore be interesting to know the probability that a set of random choices satisfying GARP also satisfies HARP, or that such a set has at 
least the same HEI. This would provide the homothetic test power conditional on GARP being satisfied. Heufer (2014a) provides an efficient method to generate sets of random choices which satisfy GARP. The conditional test power is the fraction of generated data sets that satisfies HARP.

\subsection{Experimental Data: Preferences for Giving}

Fisman et al. (2007, FKM) analyse data obtained in a laboratory experiment. They employ the same setup as Andreoni and Miller (2002, AM), that is, a generalised dictator game in which one subject (the dictator) allocates token endowments between himself and an anonymous other subject with different transfer rates. The payoffs of the dictator and the beneficiary are interpreted as two distinct goods, and the transfer rates as the price ratio. In both papers, the authors estimate a CES utility function, so they implicitly maintain the hypothesis that choices are homothetic. Testing how "close" the choices are to homotheticity is therefore important and should be conducted at least as a pretest to screen out particularly inefficient choices.

A simple two-dimensional version of homothetic efficiency has been computed for both the FKM and the AM data by Heufer (2013). We only focus on the FKM data here, as they contain 50 choices per subject as opposed to 8 in the AM data. This also allows for an informative graphical analysis based on the sets $\widetilde{H R P}$ and $\widehat{H R W}$.

We begin the analysis by calculating the AEI, HeI, Vev and Hev for all subjects. ${ }^{12}$ These results are presented in Table 1 , where each row reports the mean, minimum, the first, second (median) and third quartiles and the maximum calculated across all 76 subjects. Looking at the first two rows, we find that the AEI is noticably higher than the HeI for most subjects. However, as discussed above, the AEI and HEI are summary statistics, and may be uninformative in describing the entire distribution of the indices. In fact, looking at the third and fourth rows of Table 1, which provides the results for the VEV and HEV, gives a different picture. These results show that homothetic efficiency is, in fact, close to utility maximisation efficiency. ${ }^{13}$ In particular, the HEv displays the same pattern

\footnotetext{
${ }^{12}$ For each subject, the HEv is computed by solving the problem (19). To aid comparisons with the VEV we calculated the VEV in the L0-norm by slightly modifying the problem (6). This corresponds to how the HEv is computed and is achieved by first log-linearising the constraints (c.i)-(c.vi) and then maximise $\sum_{i=1}^{N} \tilde{v}_{i}$ where $\tilde{v}_{i}=\log \left(v_{i}\right)$. We also computed the VEV in the L1norm by solving the problem (6). Interestingly, we obtained practically identical solutions which suggests that computing the indices in the L0-norm, in fact, provides a very good approximation to the L1-problem (see the discussion in Section 3.2).

${ }^{13}$ The entries for the $\mathrm{VEV}$ and $\mathrm{HEV}$ in the third and fourth rows are averages across all subjects. For example, to obtain the entry minimum we first computed $\min \left\{v_{1}, \ldots, v_{N}\right\}$ and
} 
as the VEV: they are both characterised by one or a few observations with lower values, while the remaining values in the vector are very close to one.

\begin{tabular}{ccccccc}
\hline \multicolumn{7}{c}{ EFFICIENCY } \\
\hline Index & mean & min & 1st quartile & median & 3rd quartile & $\max$ \\
\hline AEI & 0.9407 & 0.5308 & 0.9173 & 0.9775 & 0.9972 & 1.0000 \\
HEI & 0.8821 & 0.4438 & 0.8118 & 0.9210 & 0.9786 & 1.0000 \\
VEV & 0.9959 & 0.9350 & 0.9982 & 1.0000 & 1.0000 & 1.0000 \\
HEV & 0.9788 & 0.8274 & 0.9742 & 0.9919 & 0.9986 & 1.0000 \\
\hline \hline
\end{tabular}

Table 1: Efficiency indices (FKM).

Table 2 report summary statistics for the unconditional power calculations. We present the power of GARP and HARP for different configurations, depending on how total expenditure is adjusted when applying these test procedures (see the second column). The results from the last two rows show that GARP and HARP have optimal power even when total expenditure is deflated by the VEV and HEv, respectively. Thus, we can conclude that GARP and HARP have high discriminatory power even for income-adjusted random data. However, as seen from the third and fourth row, this is not always the case when deflating expenditure by the AEI and HEI. In fact, the loss in power can be rather considerable for some subjects as seen from the third and fourth rows in Table 2. Finally, Table 3 reports the conditional power results. These results show that HARP has optimal power against uniformly random data that satisfies GARP.

\begin{tabular}{lccccccc}
\hline \multicolumn{7}{c}{ UnCONDITIONAL POWER } \\
\hline \multirow{2}{*}{ Axiom } & $\begin{array}{c}\text { Income } \\
\text { deflated }\end{array}$ & mean & min & 1st quartile & median & 3rd quartile & $\max$ \\
\hline GARP & No & 1.0000 & 1.0000 & 1.0000 & 1.0000 & 1.0000 & 1.0000 \\
HARP & No & 1.0000 & 1.0000 & 1.0000 & 1.0000 & 1.0000 & 1.0000 \\
\hline GARP & AEI & 0.9759 & 0.2520 & 1.0000 & 1.0000 & 1.0000 & 1.0000 \\
HARP & HEI & 0.9810 & 0.3720 & 1.0000 & 1.0000 & 1.0000 & 1.0000 \\
\hline GARP & VEV & 1.0000 & 1.0000 & 1.0000 & 1.0000 & 1.0000 & 1.0000 \\
HARP & HEV & 1.0000 & 1.0000 & 1.0000 & 1.0000 & 1.0000 & 1.0000 \\
\hline \hline
\end{tabular}

Table 2: Unconditional power for GARP and HARP (FKM).

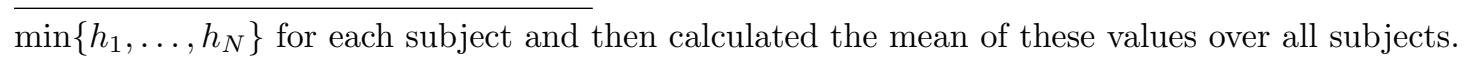




\begin{tabular}{lcccccc}
\hline \multicolumn{7}{c}{ CONDITIONAL POWER } \\
\hline Axiom & mean & min & 1st quartile & median & 3rd quartile & $\max$ \\
\hline HARP & 1.0000 & 1.0000 & 1.0000 & 1.0000 & 1.0000 & 1.0000 \\
\hline \hline
\end{tabular}

Table 3: Conditional power for HARP (FKM).

Finally, we show how our methods can recover detailed information about subjects' preferences. Figure 4 shows examples of subjects who reveal almost prototypical preferences. The differences between the homothetically revealed preferred and worse sets and the regular revealed preferred and worse sets demonstrate how much more we can learn about subjects' preferences when deviations from homotheticity are minor. The examples also illustrate that the theoretical problem of disagreements between $R P$ and HRP described in Section 3.3 is unlikely to occur for large sets of real data. The figure is a nice illustration of the extend of additional information recovered. Given that the bound on the money metric utility function is based on the revealed preferred set, the figure shows how much tighter the bounds can be when using homothetically revealed preference.

We also provide an interactive application prepared with Wolfram Mathematica ${ }^{\circledR}$ that allows users to analyse the data graphically and create figures as the ones in Figure 4 for arbitrary subjects and bundles. This software is available on one of the authors' websites and can be run with the free Wolfram CDF Player. ${ }^{14}$

\subsection{Survey Data: Household Expenditures}

We now illustrate our methods using data from the Spanish Continuous Family Expenditure Survey (Encuesta Continua de Presupuestos Familiares, abbreviated ECPF). This data was obtained from Crawford (2010) and is a quarterly budget survey, ranging from 1985-1997, that interviews Spanish households for up to a maximum of eight consecutive quarters on their consumption expenditures. ${ }^{15}$ From this data, we use a sub-sample of couples with and without children, where the husband is employed full-time and the wife is outside the labor force. ${ }^{16}$ We exclude durable goods and focus exclusively on consumption expenditures on non-durable

\footnotetext{
${ }^{14}$ The CDF file is available at https://sites.google.com/site/janheufer/ HomotheticRecoverability.cdf. To run the file, the free Wolfram CDF Player can be obtained at http://www. wolfram.com/cdf-player/.

${ }^{15}$ The data can be downloaded from the supplementary material accompanying Crawford (2010). See also Browning and Collado (2001) for a detailed discussion of the data.

${ }^{16}$ Households are randomly rotated at a rate of 12.5 percent per quarter.
} 


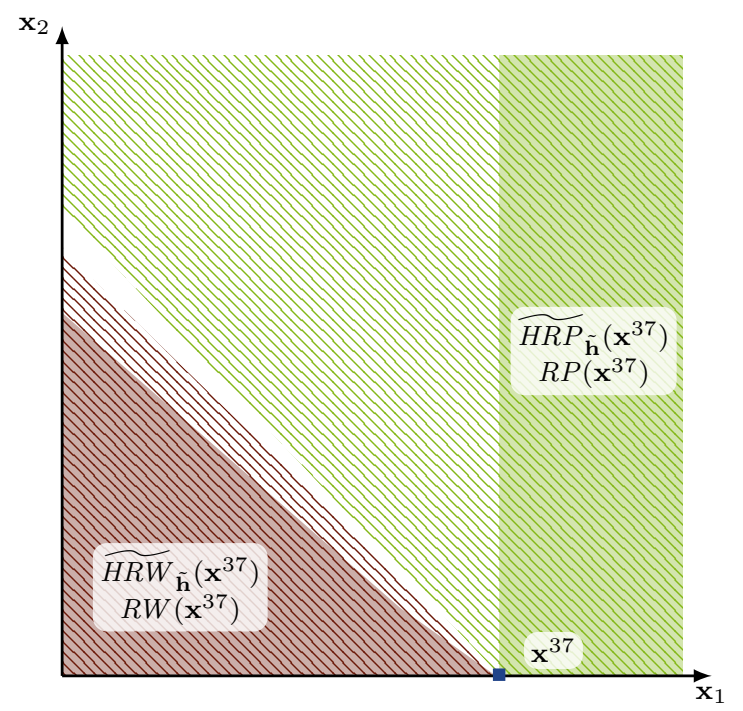

(a)

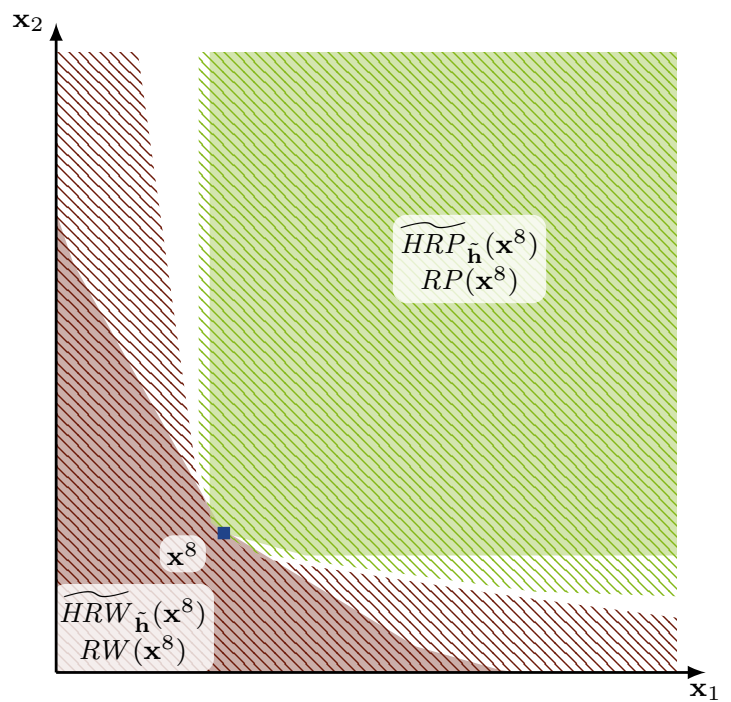

(c)

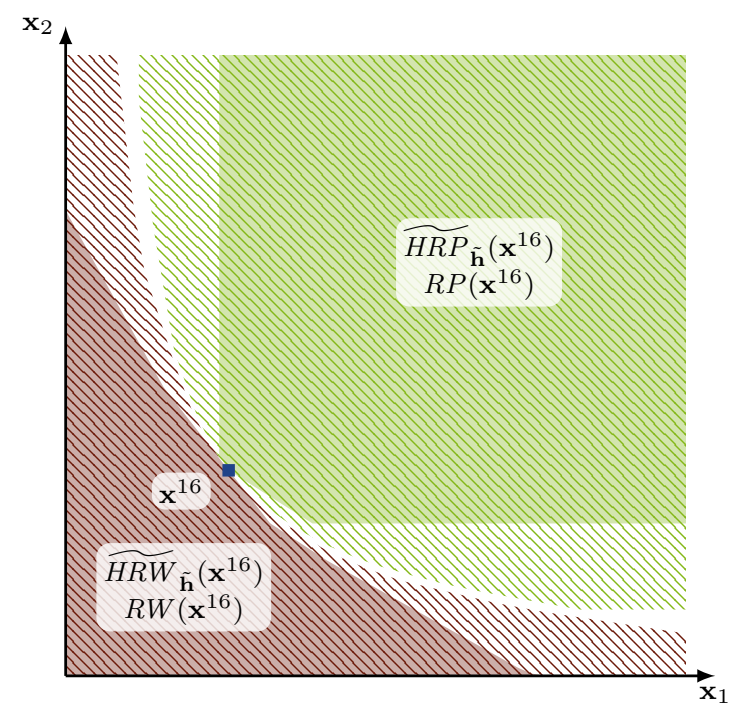

(b)

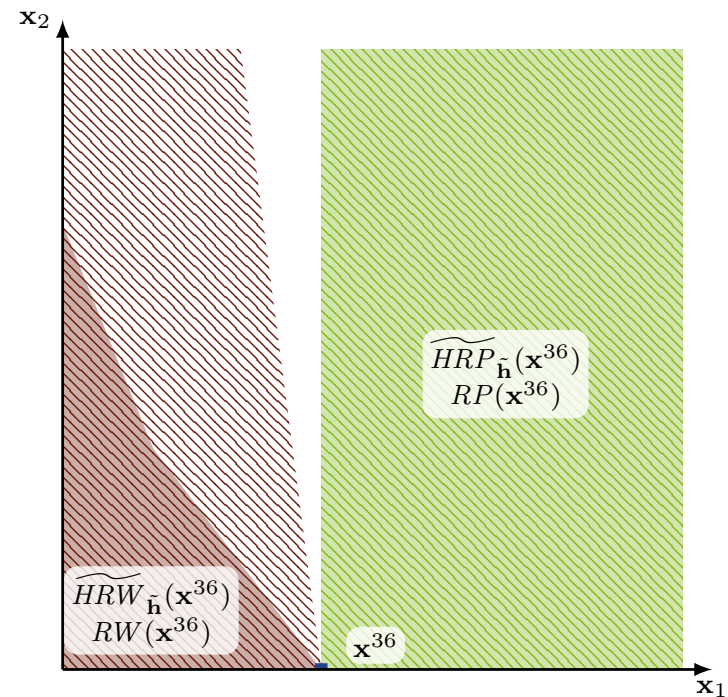

(d)

Figure 4: The dashed area shows $\widetilde{H R P}$ and $\widetilde{H R W}$, the filled area shows $R P$ and $R W$. (a) Subject 18: Weak perfect substitute preferences. (b) Subject 26: Weak Nash preferences. (c) Subject 25: Weak Rawlsian preferences. (d) Subject 19: Selfish preferences. 
consumption categories. ${ }^{17}$ Overall, we use data containing 21,866 observations on 3,134 households.

Table 4 reports summary statistics for the calculated efficiency indices across all households. As seen from this table, homothetic efficiency is very close to utility maximisation efficiency. For example, the mean across all observations and households of the HEV is 0.9960 (compared to the VEV which is 1.0000). Thus, the consumption choices of the households' seem to be very well explained by homothetic preferences.

\begin{tabular}{ccccccc}
\hline \multicolumn{7}{c}{ EFFICIENCY } \\
\hline Index & mean & min & 1st quartile & median & 3rd quartile & $\max$ \\
\hline AEI & 0.9998 & 0.9698 & 1.0000 & 1.0000 & 1.0000 & 1.0000 \\
HEI & 0.9917 & 0.9518 & 0.9890 & 0.9936 & 0.9965 & 1.0000 \\
VEV & 1.0000 & 0.9998 & 1.0000 & 1.0000 & 1.0000 & 1.0000 \\
HEV & 0.9960 & 0.9865 & 0.9936 & 0.9978 & 0.9996 & 1.0000 \\
\hline \hline
\end{tabular}

Table 4: Efficiency indices (ECPF).

Table 5 report results from the power analysis. As discussed in the Introduction, allowing for errors in revealed preference tests often lead to a loss in power, which may render the analysis practically meaningless. And as seen from Table 5, this concern is clearly warranted. Indeed, the first, third and fifth rows show that standard utility maximisation barely has any power against uniformly random behaviour. This means that GARP is essentially unable to reject random consumption behaviour. On the other hand, because HARP is a stronger condition than GARP, we expect it to have higher power against random behaviour. The second, fourth and sixth rows show that HARP indeed has substantially more power against uniformly random behaviour than GARP. In fact, while the average power across households never exceeds nine percent for GARP, the power of HARP is above 90 percent for all but a few households.

The current analysis also allow us to analyse the potential loss in power of adjusting expenditure by efficiency in revealed preference tests. Consider once again the second, fourth and sixth rows in Table 5. These results show that while the loss in power can be considerable when adjusting income by the HEI, the loss in power is negligible when adjusting income by the HEv. More precisely, adjusting

\footnotetext{
${ }^{17}$ The non-durables are aggregated into the following 15 consumption categories: (i) food and non-alcoholic drinks at home, (ii) alcohol, (iii) tobacco, (iv) energy at home, (v) services at home, (vi) non-durables at home, (vii) non-durable medicines, (viii) medical services, (ix) transportation, (x) petrol, (xi) leisure, (xii) personal services, (xiii) personal non-durs, (xvi) restaurants and bars, and $(\mathrm{xv})$ travelling.
} 


\begin{tabular}{lccccccc}
\hline \multicolumn{7}{c}{ UnCONDITIONAL POWER } \\
\hline \multirow{2}{*}{ Axiom } & $\begin{array}{c}\text { Income } \\
\text { deflated }\end{array}$ & mean & min & 1st quartile & median & 3rd quartile & $\max$ \\
\hline GARP & No & 0.0892 & 0.0000 & 0.0000 & 0.0280 & 0.1700 & 0.7080 \\
HARP & No & 0.9974 & 0.9700 & 0.9980 & 1.0000 & 1.0000 & 1.0000 \\
\hline GARP & AEI & 0.0859 & 0.0000 & 0.0000 & 0.0260 & 0.1640 & 0.6480 \\
HARP & HEI & 0.6382 & 0.0000 & 0.3760 & 0.7400 & 0.9280 & 1.0000 \\
\hline GARP & VEV & 0.0877 & 0.0000 & 0.0000 & 0.0260 & 0.1680 & 0.6760 \\
HARP & HEV & 0.9558 & 0.1300 & 0.9480 & 0.9840 & 0.9960 & 1.0000 \\
\hline \hline
\end{tabular}

Table 5: Unconditional power for GARP and HARP (ECPF).

income by the HEv have small effects on the power, which, in our view, rather forcefully addresses the concern that adjusting expenditure by efficiency in revealed preference testing renders the analysis meaningless.

Table 6 reports the conditional power results. These results show that HARP has very good power against uniformly random data that satisfies GARP.

\begin{tabular}{lcccccc}
\hline \multicolumn{7}{c}{ CONDITIONAL POWER } \\
\hline Axiom & mean & min & 1st quartile & median & 3rd quartile & $\max$ \\
\hline HARP & 0.9776 & 0.7400 & 0.9800 & 0.9900 & 1.0000 & 1.0000 \\
\hline \hline
\end{tabular}

Table 6: Conditional power for HARP (ECPF).

The main conclusions that can be drawn from our results so far are that: (i) The consumption choices of the households are very close to satisfying homothetic preferences (i.e., the households have high homothetic efficiency) and (ii) Homotheticity has much higher discriminatory power against random behaviour than standard utility maximisation. In fact, the vast majority of households have optimal power against random behaviour even when income is adjusted by homothetic efficiency. But this is a sequential analysis, and as such, fails to give any indication of the trade-off between efficiency and power. Beatty and Crawford (2011) and Heufer $(2012)^{18}$ suggested to combine efficiency and power into one single measure based on the idea of predictive success originally put forward by Selten (1991). Beatty and Crawford's (2011) measure is computed as the difference between pass rate and one minus the power. The outcome of this measure is a value between minus one and

\footnotetext{
${ }^{18}$ See also Heufer (2008) for an early application of the trade-off approach to compare two different efficiency indices.
} 
one. Negative values would suggest that the model fails to describe the preferences of the household: The model does not pass the revealed preference axiom and provides low discriminatory power against random behaviour. In contrast, a high and positive predictive success indicates a potentially useful model: It passes the revealed preference axiom and has high power against random behaviour.

As suggested by Beatty and Crawford (2011) and Heufer (2012), the predictive success can be used to find the optimal level of efficiency. Suppose we want to find the optimal efficiency level for HARP at the household level. For a given efficiency level, we first calculate $\operatorname{HARP}(e)$ which gives the pass rate (either zero or one) and then, in a second, we calculate the power using $\operatorname{HARP}(e)$. Doing this for an arbitrarily fine grid gives the predictive success at each efficiency level. The optimal efficiency level is the one that produces the highest predictive success. Figure 5 presents the results from this analysis. Figure 5.(a) shows the average predictive success across all households for each efficiency level in the grid. As seen from this plot, GARP obtains the maximal predictive success value at an efficiency level of one where it is very close to zero. One interpretation of this is that the theory of standard utility maximisation performs about as well as a theory that explains consumer demand as purely random behaviour. The results for HARP are quite different: We find that the maximal average predictive success across households is 0.22 obtained at an efficiency level of 0.995 . Thus, according to these results, homotheticity provides a considerably better fit to the data than standard utility maximisation at an efficiency level only slightly below one. Figures 5.(b)-(d) show the median and the first and third quartile of the predictive success across all households for each efficiency level. These plots show that the predictive success is always higher for HARP at some efficiency level equal to or above 0.985. Focusing on Figure 5.(b), we find that half of all households have a predictive success above 0.27 at efficiency level 0.99. Finally, looking at plot Figure 5.(d), we see that $25 \%$ of all households have a predictive success above 0.74 at efficiency level 0.99 . Assuming that an efficiency level of 0.985 is acceptable, we can then draw the conclusion, based on the measure of predictive success, that homotheticity is a more suitable model to describe the preferences of the households in this survey data.

\section{Conclusion}

Consumer choice data often violates homothetic utility maximisation. In such cases, it would be interesting to know how close the data comes to homothetic utility maximisation. For this purpose, we introduced a non-parametric approach to estimating homothetic efficiency of demand data by generalising Heufer's (2013) method. We introduced the Homothetic Efficiency Index (HEI) and the Homothetic 


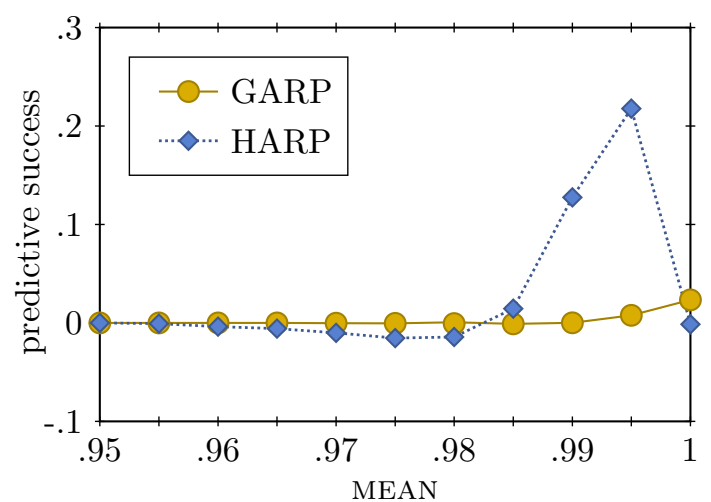

(a)

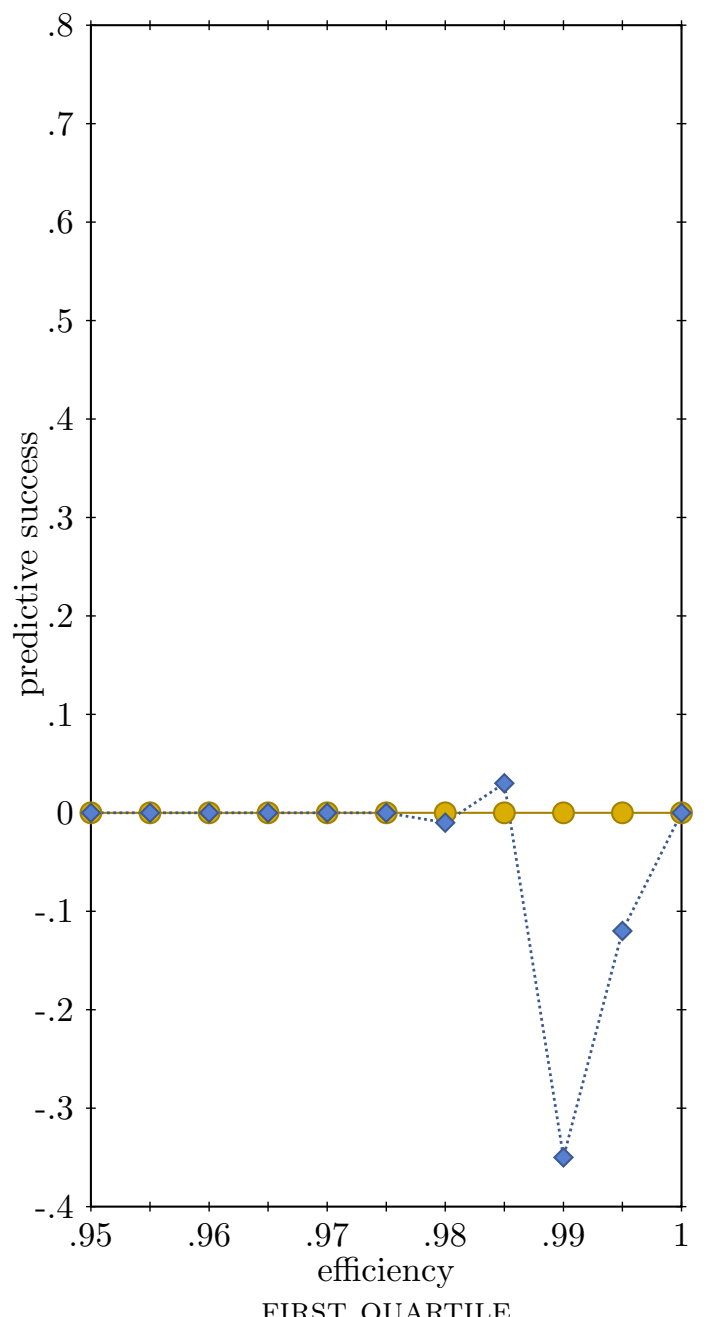

(c)

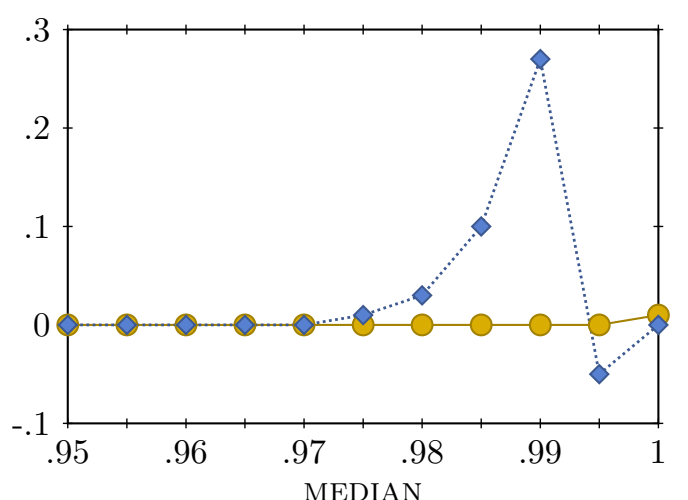

(b)

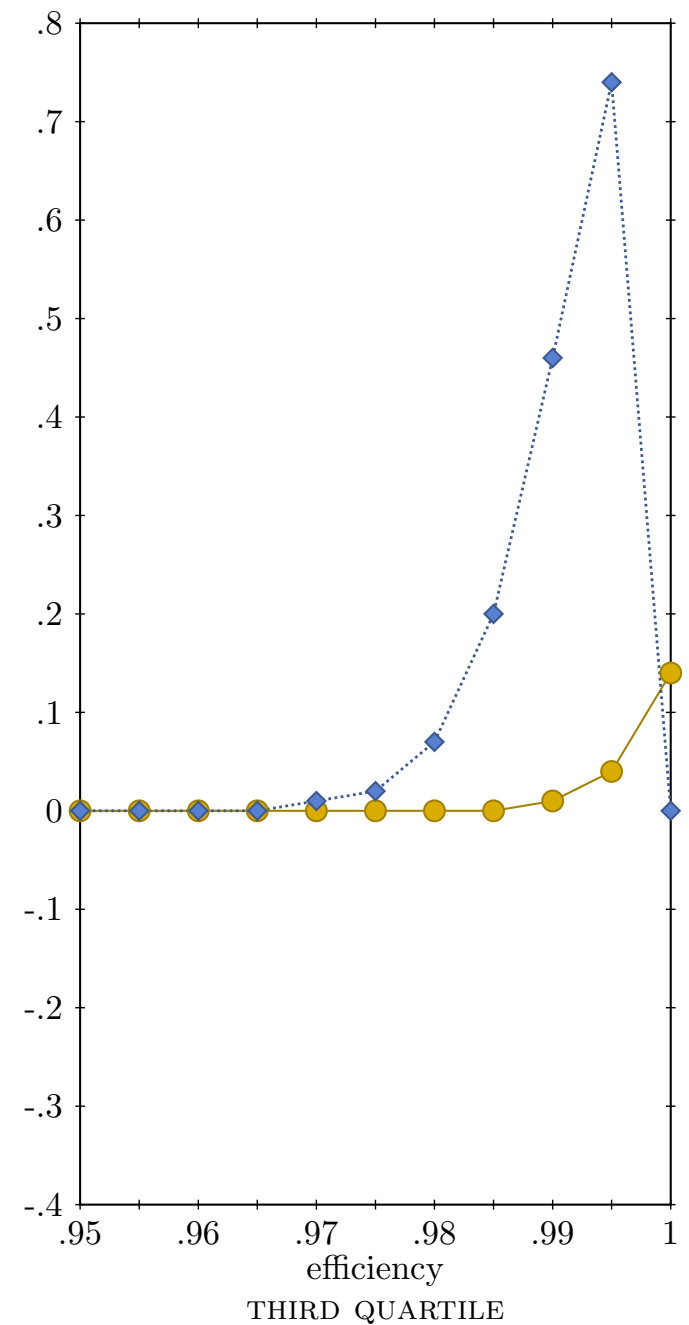

(d)

Figure 5: Measure of predictive success across all households for each efficiency level in the grid $(\mathrm{ECPF})$. 
Efficiency Vector (HEv) in analogy to the standard Afriat Efficiency Index (AEI) and Varian's improved violation index or Varian Efficiency Vector (VEV). As the AEI, the HEI can be interpreted as a measure of wasted income under the assumption that violations of homotheticity were due to errors in decision making. As a non-parametric approach, our method does not rely on any specific form of a utility function.

Both the HEI and the HEv can be used to adjust data by deflating expenditure to reconstruct bounds on preferred and worse sets. This is motivated by a concept called $e$ - and $\mathbf{h}$-rationalisation which is similar to a concept used recently by Halevy et al. (2012): For efficiency close to $100 \%$, there still exists a utility function that adequately explains the data as the result of homothetic utility maximisation with minor errors.

We applied the method to two data sets. This empirical analysis illustrates how recoverability based on adjusted homothetically revealed preferred relations allows a detailed analysis of preferences at the individual level. It also demonstrates how a data set that has very low power against the alternative hypothesis of random behaviour can still be useful when testing for the stronger condition of homothetic utility maximisation. We find that efficiency can be very high for homotheticy, that tests for HARP have far greater power than tests for GARP, and that adjusting choices by efficiency measures has negligible effects on test power. Using the measure of predictive success applied to households consumer data, we find that homothetic utility maximisation is considerably more successful in explaining the demand behaviour for efficiency levels close to one.

We expect that our results help in analysing experimental, survery, and field data. It will help to test the assumption of homotheticity before estimating homothetic utility functions, to quantify the extent of the violation of homotheticity, to analyse preferences in detail without the need of estimating parameters, and to increase test power for data sets which have too litle power against the alternative hypothesis of random behaviour.

The approach could easily be translated to production analysis. As homotheticity of production is assumed in many applications, a non-parametric test that provides a measure for homothetic efficiency independent of a specific production function should at the very least be a useful screening device and robustness check before parameters of a homothetic production function are estimated. Nonparametric recoverability of technological information as suggested by Varian (1984a) could also be carried out in analogy to the recoverability of preferences in our paper. 


\section{A Appendix}

\section{A.1 Proofs}

\section{A.1.1 Proof of Theorem 4}

Proof For $(1) \Rightarrow(2)$ and $(3) \Rightarrow(1)$, Varian's (1983) proof can be applied with minor obvious adjustments. We will prove $(2) \Rightarrow(3)$.

As in Varian (1983), define $U(\mathbf{x})=\min _{i}\left\{U^{i} \mathbf{p}^{i} \mathbf{x}\right\}$. It can be easily verified that $U \in \mathcal{U}$ and that $U$ is homothetic; what remains to be shown is that $U$ e-rationalises $\Omega$. Suppose $\Omega$ satisfies $\operatorname{HARP}(e)$ and there exists $\mathbf{x}$ such that $U(\mathbf{x}) \geq U\left(\mathbf{x}^{i}\right)$ and $\mathbf{x}^{i} \mathrm{P}^{0}(e) \mathbf{x}$. Then $e \mathbf{p}^{i} \mathbf{x}^{i}=e>\mathbf{p}^{i} \mathbf{x}$. By continuity and monotonicity of $U$, there then exists $\mathbf{y}>\mathbf{x}$ such that $\mathbf{p}^{i} \mathbf{y}=e$ and $U(\mathbf{y})>U(\mathbf{x}) . \operatorname{By} \operatorname{Harp}(e)$, $e U^{i} \leq \min _{j}\left\{U^{j} \mathbf{p}^{j} \mathbf{x}^{i}\right\}$, and with $\mathbf{p}^{i} \mathbf{y}=e$ we obtain $\mathbf{p}^{i} \mathbf{y} U^{i} \leq \min _{j}\left\{U^{j} \mathbf{p}^{j} \mathbf{x}^{i}\right\}$. Suppose $U\left(\mathbf{x}^{i}\right)=U^{k} \mathbf{p}^{k} \mathbf{x}^{i}$; then $\mathbf{p}^{i} \mathbf{y} U^{i} \leq U^{k} \mathbf{p}^{k} \mathbf{x}^{i}=U\left(\mathbf{x}^{i}\right) \leq U(\mathbf{x})$. But $U(\mathbf{y})=$ $\min _{j}\left\{U^{j} \mathbf{p}^{j} \mathbf{y}\right\}$, so $U(\mathbf{y}) \leq U^{i} \mathbf{p}^{i} \mathbf{y}$. Then $U(\mathbf{y}) \leq U\left(\mathbf{x}^{i}\right) \leq U(\mathbf{x})$, but $\mathbf{y}>\mathbf{x}$, which contradicts montonicity.

Suppose instead that there exists $\mathbf{x}$ such that $U(\mathbf{x})>U\left(\mathbf{x}^{i}\right)$, and $\mathbf{x}^{i} \mathrm{R}^{0}(e) \mathbf{x}$ but not $\mathbf{x}^{i} \mathrm{P}^{0}(e) \mathbf{x}$. Then $\mathbf{p}^{i} \mathbf{x}=e$, and we obtain $U(\mathbf{x}) \leq U\left(\mathbf{x}^{i}\right) \leq U(\mathbf{x})$, which implies $U(\mathbf{x})=U\left(\mathbf{x}^{i}\right)$, a contradiction. Thus, $U$ e-rationalises $\Omega$.

\section{A.1.2 Proof of Theorem 6}

Proof By induction. Suppose $u \in \mathcal{U}$ is homothetic and $\mathbf{h}$-rationalises the data. By definition, we can assume without loss of generality that $u$ is homogenous of degree 1. Because $u$ is concave, we only need to consider the vertices of the closure of $\widetilde{H R P}_{\mathbf{h}}$, that is, we only need to check if $u\left(\tilde{t}_{i, \diamond} \mathbf{x}^{i}\right)<u\left(\mathbf{x}^{\diamond}\right)$ is possible.

Step 1 By homogeneity of degree $1, u\left(\left[\mathbf{p}^{i} \mathbf{x}^{\diamond} / h_{i}^{2}\right] \mathbf{x}^{i}\right)=\left[\mathbf{p}^{i} \mathbf{x}^{\diamond} / h_{i}^{2}\right] u\left(\mathbf{x}^{i}\right)$. Let $\mathbf{y}=\left[h^{i} \mathbf{x}^{\diamond}\right] /\left[\mathbf{p}^{i} \mathbf{x}^{\diamond}\right]$; then $\mathbf{p}^{i} \mathbf{y}=h_{i}$, and therefore $\mathbf{x}^{i} \mathrm{R}^{0}\left(h_{i}\right) \mathbf{y}$. Suppose $u\left(\tilde{t}_{i, \diamond} \mathbf{x}^{i}\right)<$ $u\left(\mathbf{x}^{\diamond}\right)$. Then $u\left(\mathbf{x}^{i}\right)<\left(h_{i}^{2} /\left[\mathbf{p}^{i} \mathbf{x}^{\diamond}\right]\right) u\left(\mathbf{x}^{\diamond}\right)$. But with $h_{i} \leq 1,\left(h_{i}^{2} /\left[\mathbf{p}^{i} \mathbf{x}^{\diamond}\right]\right) u\left(\mathbf{x}^{\diamond}\right) \leq$ $\left(h_{i} /\left[\mathbf{p}^{i} \mathbf{x}^{\diamond}\right]\right) u\left(\mathbf{x}^{\diamond}\right)=u(\mathbf{y})$. Then $u\left(\mathbf{x}^{i}\right)<u(\mathbf{y})$, but $\mathbf{x}^{i} \mathrm{R}^{0}\left(h_{i}\right) \mathbf{y}$, so $u$ cannot $\mathbf{h}-$ rationalise $\Omega$. Thus, $\left(\left[\mathbf{p}^{i} \mathbf{x}^{\diamond}\right] / h_{i}^{2}\right) u\left(\mathbf{x}^{i}\right) \geq u\left(\mathbf{x}^{\diamond}\right)$.

Step 2 Assume without loss of generality that

$$
\tilde{t}_{1, n}=\frac{\mathbf{p}^{1} \mathbf{x}^{2}}{h_{i}^{2}} \frac{\mathbf{p}^{2} \mathbf{x}^{3}}{h_{i}^{2}} \ldots \frac{\mathbf{p}^{n-1} \mathbf{x}^{n}}{h_{n-1}^{2}}
$$

and that $\tilde{t}_{1, \diamond}=\tilde{t}_{1, n}\left[\mathbf{p}^{n} \mathbf{x}^{\diamond}\right] / h_{n}^{2}$. Suppose $\tilde{t}_{1, n} u\left(\mathbf{x}^{1}\right) \geq u\left(\mathbf{x}^{n}\right)$. Then

$$
\tilde{t}_{1, \diamond} u\left(\mathbf{x}^{1}\right)=\tilde{t}_{1, n} u\left(\mathbf{x}^{1}\right) \frac{\mathbf{p}^{n} \mathbf{x}^{\diamond}}{h_{n}^{2}} \geq u\left(\mathbf{x}^{n}\right) \frac{\mathbf{p}^{n} \mathbf{x}^{\diamond}}{e^{2}} \geq u\left(\mathbf{x}^{0}\right),
$$


where the last inequality follows from Step 1. Thus, $\tilde{t}_{1, \diamond} u\left(\mathbf{x}^{1}\right) \geq u\left(\mathbf{x}^{\diamond}\right)$.

By induction, Steps 1 and 2 show that $u\left(\tilde{t}_{i, \diamond} \mathbf{x}^{i}\right) \geq u\left(\mathbf{x}^{\diamond}\right)$ for all $i$ and that all homothetic $u \in \mathcal{U}$ which h-rationalise $\Omega$. That concludes the proof for $\widetilde{H R P}$. The second part of the theorem then follows from the definition of $\widetilde{H R W}$.

\section{References}

Afriat, S. N. (1967): "The Construction of Utility Functions From Expenditure Data," International Economic Review 8(1):67-77.

(1972): "Efficiency Estimation of Production Functions," International Economic Review 13(3):568-598.

Andreoni, J. and Miller, J. (2002): "Giving According to GARP: An Experimental Test of the Consistency of Preferences for Altruism," Econometrica 70(2):737753 .

Apesteguia, J. and Ballester, M. A. (2015): "A Measure of Rationality and Welfare," Journal of Political Economy forthcoming.

Beatty, T. K. M. and Crawford, I. A. (2011): "How Demanding Is the Revealed Preference Approach to Demand?" American Economic Review 101(6):27822795.

Becker, N., Häger, K., and Heufer, J. (2013a): "Revealed Notions of Distributive Justice I: Theory," Ruhr Economic Papers \#443, TU Dortmund University, Discussion Paper.

(2013b): "Revealed Notions of Distributive Justice II: Experimental Analysis," Ruhr Economic Papers \#444, TU Dortmund University, Discussion Paper, working paper.

Blundell, R., Browning, M., and Crawford, I. (2003): "Nonparametric Engel Curves and Revealed Preference," Econometrica 71(1):205-240.

(2008): "Best nonparametric bounds on demand responses," Econometrica 76(6):1227-1262.

Bronars, S. G. (1987): "The Power of Nonparametric Tests of Preference Maximization," Econometrica 55(3):693-698. 
Browning, M. and Collado, M. D. (2001): "The response of expenditures to anticipated income changes: panel data estimates," American Economic Review 91(3):681-692.

Camille, N., Griffiths, C. A., Vo, K., Fellows, L. K., and Kable, J. W. (2011): "Ventromedial Frontal Lobe Damage Disrupts Value Maximization in Humans," The Journal of Neuroscience 31(20):7527-7532.

Chaney, T. (2008): "Distorted gravity: The intensive and extensive margins of international trade," American Economic Review 98(1707-1721):4.

Cherchye, L., De Rock, B., Sabbe, J., and Vermeulen, F. (2008): "Nonparametric Tests of Collectively Rational Consumption Behavior: An Integer Programming Procedure," Journal of Econometrics 147(2):258-265.

Cherchye, L., Demuynck, T., De Rock, B., and Hjertstrand, P. (2015): "Revealed Preference Tests for Weak Separability: An Integer Programming Approach," Journal of Econometrics 186:129-141.

Chipman, J. S. (1965): "A Survey of the Theory of International Trade: Part 2, The Neo-Classical Theory," Econometrica 33(4):685-760.

- (1974): "Homothetic Preferences and Aggregation," Journal of Economic Theory 8(1):26-38.

Choi, S., Fisman, R., Gale, D., and Kariv, S. (2007a): "Consistency and Heterogeneity of Individual Behavior under Uncertainty," American Economic Review 97(5):1921-1938.

(2007b): "Revealing Preferences Graphically: An Old Method Gets a New Tool Kit," American Economic Review 97(2):153-158.

Cox, J. (1997): "On Testing the Utility Hypothesis," The Economic Journal 107:1054-1078.

Crawford, I. (2010): "Habits revealed," The Review of Economic Studies 77(4):13821402 .

Dean, M. and Martin, D. (2015): "Measuring Rationality with the Minimum Cost of Revealed Preference Violations," Working paper.

Dickinson, D. L. (2009): "Experiment Timing and Preferences for Fairness," The Journal of Socio-Economics 38(1):89-95. 
Diewert, W. E. (1973): "Afriat and Revealed Preference Theory," Review of Economic Studies 40(3):419-425.

Echenique, F., Lee, S., and Shum, M. (2011): "The Money Pump as a Measure of Revealed Preference Violations," Journal of Political Economy 119(6):12011223 .

Eisenberg, E. (1961): "Aggregation of Utility Functions," Management Science $7(4): 337$.

Février, P. and Visser, M. (2004): "A Study of Consumer Behavior Using Laboratory Data," Experimental Economics 7(1):93-114.

Fisman, R., Kariv, S., and Markovits, D. (2005): "Distinguishing Social Preferences from Preferences for Altruism," Institute for Advanced Study, Economics Working Papers 0061.

(2007): "Individual Preferences for Giving," American Economic Review 97(5):1858-1876.

Gorman, W. M. (1953): "Community Preference Fields," Econometrica 21(1):6380 .

(1959): "Separable utility and aggregation," Econometrica 27(3):469-481.

Gross, J. (1995): "Testing Data for Consistency with Revealed Preference," Review of Economics and Statistics 77(4):701-710.

Halevy, Y., Persitz, D., and Zrill, L. (2012): "Parametric Recoverability of Preferences," Working paper.

Hanoch, G. and Rothschild, M. (1972): "Testing the Assumptions of Production Theory: A Nonparametric Approach," Journal of Political Economy $80(2): 256-275$.

Harbaugh, W. T., Krause, K., and Berry, T. R. (2001): "GARP for Kids: On the Development of Rational Choice Behavior," American Economic Review 91(5):1539-1545.

Helpman, E., Melitz, M., and Rubinstein, Y. (2008): "Estimating trade flows: Trading partners and trading volumes," Quarterly Journal of Economics 123(2):441-487.

Heufer, J. (2008): "A Geometric Measure for the Violation of Utility Maximization," Ruhr Economic Papers \#69, TU Dortmund University, Discussion Paper. 
(2012): "Testing for Utility Maximization with Error and the Loss of Power," German Economic Review 13(2):161-173.

(2013): "Testing Revealed Preferences for Homotheticity with Two-Good Experiments," Experimental Economics 16(1):114-124.

(2014a): "Generating Random Optimising Choices," Computational Economics 44(3):295-305.

(2014b): "Nonparametric Comparative Revealed Risk Aversion," Journal of Economic Theory 153:569-616.

Heufer, J. and Hjertstrand, P. (2015): "Consistent subsets: Computationally feasible methods to compute the Houtman-Maks-Index," Economics Letters 128:87-89.

Houtman, M. and Maks, J. (1985): "Determining all Maximal Data Subsets Consistent with Revealed Preference," Kwantitatieve Methoden 19:89-104.

- (1987): The Existence of Homothetic Utility Functions Generating Dutch Consumer Data, University of Groningen, Groningen.

Knoblauch, V. (1992): "A Tight Upper Bound on the Money Metric Utility Function," American Economic Review 82(3):660-663.

- (1993): "Recovering Homothetic Preferences," Economics Letters 43(1):4145 .

Krugman, P. (1980): "Scale economics, product differentiation, and the pattern of trade," American Economic Review 70(5):950-959.

Liu, P.-W. and Wong, K.-C. (2000): "Revealed Homothetic Preference and Technology," Journal of Mathematical Economics 34(3):287-314.

Manser, M. E. and McDonald, R. J. (1988): "An analysis of substitution bias in measuring inflation, 1959-85," Econometrica 56(4):909-930.

Mantel, R. R. (1976): "Homothetic Preferences and Community Excess Demand Functions," Journal of Economic Theory 12(2):197-201.

Mattei, A. (2000): "Full-scale real tests of consumer behavior using experimental data," Journal of Economic Behavior \& Organization 43(4):487-497.

Melitz, M. J. (2003): "The impact of trade on intra-industry reallocations and aggregate industry productivity," Econometrica 71(1695-1725):6. 
Polemarchakis, H. M. (1983): "Homotheticity and the Aggregation of Consumer Demands," Quarterly Jo 98(2):363-369.

Selten, R. (1991): "Properties of a Measure of Predictive Success," Mathematical Social Sciences 21(2):153-167.

Shafer, W. and Sonnenschein, H. (1982): "Market Demand and Excess Demand Functions," K. J. Arrow and M. D. Intriligator (Editors), "Handbook of Mathematical Economics," volume 2, 671-693, Amsterdam: North-Holland.

Silva, E. and Stefanou, S. E. (1996): "Generalization of Nonparametric Tests for Homothetic Production," American Journal of Agricultural Economics 78(3):542-546.

Sippel, R. (1997): "An Experiment on the Pure Theory of Consumer's Behavior," The Economic Journal 107(444):1431-1444.

Varian, H. R. (1982): "The Nonparametric Approach to Demand Analysis," Econometrica 50(4):945-972.

(1983): "Non-parametric Tests of Consumer Behaviour," Review of Economic Studies 50(1):99-110.

(1984a): "The Nonparametric Approach to Production Analysis," Econometrica 52(3):579-597.

(1984b): "Social Indifference Curves and Aggregate Demand," Quarterly Journal of Economics 99(3):403-414.

(1990): "Goodness-of-Fit in Optimizing Models," Journal of Econometrics 46(1-2):125-140.

(1993): "Goodness-of-Fit for Revealed Preference Tests," Working Paper, University of Michigan. 